\title{
Power Allocation for Secondary Outage Minimization in Spectrum Sharing Networks with Limited Feedback
}

\author{
YuanYuan He and Subhrakanti Dey, Senior Member, IEEE
}

\begin{abstract}
We address an optimal transmit power allocation problem that minimizes the outage probability of a secondary user (SU) who is allowed to coexist with a primary user (PU) in a narrowband spectrum sharing cognitive radio network, under a long term average transmit power constraint at the secondary transmitter (SU-TX) and an average interference power constraint at the primary receiver (PU-RX), with quantized channel state information (CSI) (including both the channels from SU-TX to SU-RX, denoted as $g_{1}$ and the channel from SU-TX to PU-RX, denoted as $g_{0}$ ) at the SU-TX. The optimal quantization regions in the vector channel space is shown to have a "stepwise" structure. With this structure, the above outage minimization problem can be explicitly formulated and solved by employing the KarushKuhn-Tucker (KKT) necessary optimality conditions to obtain a locally optimal quantized power codebook. A low-complexity near-optimal quantized power allocation algorithm is derived for the case of large number of feedback bits. More interestingly, we show that as the number of partition regions approaches infinity, the length of interval between any two adjacent quantization thresholds on the $g_{0}$ axis is asymptotically equal when the average interference power constraint is active. Similarly, we show that when the average interference power constraint is inactive, the ratio between any two adjacent quantization thresholds on the $g_{1}$ axis becomes asymptotically identical. Using these results, an explicit expression for the asymptotic SU outage probability at high rate quantization (as the number of feedback bits goes to infinity) is also provided, and is shown to approximate the optimal outage behavior extremely well for large number of bits of feedback via numerical simulations. Analysis on the extension to multiple secondary users case (cognitive multipleaccess network) is also discussed. Numerical results illustrate that with only a few bits of feedback, the derived algorithms provide secondary outage performance very close to that with full CSI at the SU-TX.
\end{abstract}

Index Terms-Cognitive radio, spectrum sharing, outage probability, limited feedback.

\section{INTRODUCTION}

$\mathbf{S}^{\mathrm{c}}$ CARCITY of available vacant spectrum is limiting the growth of wireless products and services [1]. Traditional spectrum licensing policy forbids unlicensed users to transmit in order to avoid unfavorable interference at the cost of spectral utilization efficiency. This led to the idea of cognitive

Manuscript received July 3, 2011; revised August 13, 2012 and November 27,2012 . The editor coordinating the review of this paper and approving it for publication was N. Al-Dhahir.

The authors are with the Department of Electrical and Electronic Engineering, University of Melbourne, Victoria 3010, Australia (e-mail: \{yuhe, sdey\}@unimelb.edu.au).

This paper was partly presented at the Australian Communications Theory Workshop 2012 [33].

Digital Object Identifier 10.1109/TCOMM.2013.043013.110431 radio (CR) technology, originally introduced by J. Mitola [2], which holds tremendous promise to dramatically improve the efficiency of spectral utilization.

The key idea behind CR is that an unlicensed/secondary user (SU) is allowed to communicate over a frequency band originally licensed to a primary user (PU), as long as the transmission of SU does not generate unfavorable impact on the operation of PU in that band. Effectively, three categories of CR network paradigms have been proposed: interweave, overlay, and underlay [3]. In the underlay systems, also known as spectrum sharing model, which is the focus of this paper, the SU can transmit even when the PU is present, but the transmit power of SU should be controlled properly so as to ensure that the resulting interference does not degrade the received signal quality of PU to an undesirable level [4] by imposing the so called interference temperature [5] constraints at PU (average or peak interference power (AIP/PIP) constraint) and as well as to enhance the performance of SU transmitter (SUTX) to SU receiver (SU-RX) link.

Various notions of capacity for wireless channels include ergodic capacity (for delay-insensitive services), delay-limited capacity and outage probability (for real-time applications). These information theoretic capacity notions constitute important performance measures in analyzing the performance limits of CR systems. In [5], the authors investigated the ergodic capacity of such a dynamic narrowband spectrum sharing model under either AIP or PIP constraint at PU receiver (PU-RX) in various fading environments. The authors of [6] extended the work in [5] to asymmetric fading environments. In [7], the authors studied optimum power allocation for three different capacity notions under both AIP and PIP constraints. In [4], the authors also considered the transmit power constraint at the SU-TX and investigated the optimal power allocation strategies to achieve the ergodic capacity and outage capacity of SU under various combinations of secondary transmit (peak/average) power constraints and interference (peak/average) constraints. The optimal power allocation schemes for achieving the ergodic sum capacity of the fading cognitive multiple-access channel and cognitive broadcast channel were first studied in [29]. [28] investigated the outage minimization problem under interference power constraint together with the individual transmit power constraint of each SU for fading cognitive multiple-access channels. In [30], power control algorithms were provided for a cognitive radio network consisting of primary and secondary 
users as a multiple-access system. In [31], the authors jointly designed power control, scheduling and routing for multi-hop cognitive radio networks.

Achieving the optimal system performance for a SU requires the SU-TX to acquire full channel state information (CSI) including the channel information from SU-TX to PURX and the channel information from SU-TX to SU-RX. Most of the above results assume perfect knowledge or full CSI, which is very difficult to implement in practice, especially the channel information from SU-TX to PU-RX without PU's cooperation. A few recent papers have emerged that address this concern by investigating performance analysis with various forms of partial CSI at SU-TX, such as noisy CSI and quantized CSI. With assumption of perfect knowledge of the CSI from SU-TX to SU-RX channel, [8] studied the effect of imperfect channel information of the SU-TX to PURX channel under AIP or PIP constraint by considering the channel information from SU-TX to the PU-RX as a noisy estimate of the true CSI and employing the so-called 'tifr' transmission policy. Another recent work [9] also considered imperfect CSI of the SU-TX to PU-RX channel in the form of noisy channel estimate (a range from near-perfect to seriously flawed estimates) and studied the effect of using a midrise uniformly quantized CSI of the SU-TX to PU-RX channel, while also assumed the SU-TX had full knowledge of the CSI from SU-TX to SU-RX channel. Recently, [10] has proposed a practical design paradigm for cognitive beamforming based on finite-rate cooperative feedback from the PU-RX to the SU-TX and cooperative feedforward from the SU-TX to the PU-RX. A robust cognitive beamforming scheme was also analyzed in [11], where full channel information on SU-TX to SU-RX channel was assumed, and the imperfect channel information on the SU-TX to PU-RX channel was modelled using an uncertainty set. Finally, [12] studied the issue of channel quantization for resource allocation via the framework of utility maximization in OFDMA based CR networks, but did not investigate the joint channel partitioning and rate/power codebook design problem. The absence of a rigorous and systematic design methodology for quantized resource allocation algorithms in the context of cognitive radio networks motivated our earlier work [13], where we addressed an SU ergodic capacity maximization problem in a wideband spectrum sharing scenario with quantized information about the vector channel space involving the SU-TX to SU-RX channel and the SU-TX to PU-RX channel over all bands, under an average transmit power constraint at the SU-TX and an average interference constraint at the PU-RX. [14] studied the spectral efficiency maximization problem based on the quantized CSI sent from SU-RX and PU-RX separately to the SU-TX. A slightly different approach was taken in [15], [16] where the SU overheard the PU feedback link information and used this to obtain information about whether or not the PU is in outage and how the SU-TX should control its power to minimize interference on the PU-RX.

In this paper, we address the problem of minimizing the SU outage probability under an average transmit power (ATP) constraint at the SU-TX and an average interference power (AIP) constraint at the PU-RX. Similar to [13], we consider an infrastructure-based narrowband spectrum sharing scenario where an SU communicates to its receiver contained within the SU base station (SU-BS) on a narrowband channel shared with a PU communicating to its receiver, located within the primary base station (PU-BS). The key problem involves a joint design of the optimal partition regions of the vector channel space (consisting of the SU-TX to SU-BS channel (denoted by power gain $g_{1}$ ) and the interfering channel between the SUTX and PU-BS (denoted by power gain $g_{0}$ )) and the design of the corresponding optimal power codebook. This is solved offline at a central controller called the CR network manager as in [13], based on the channel statistics. We assume that the CR network manager is able to obtain full CSI information of the vector channel space $\left(g_{1}, g_{0}\right)$ in real-time from the SUBS and PU-BS, respectively, via optical-fibre links (similar to backhaul links in multi-cell MIMO cooperative networks [17] or coordinated multipoint (COMP) networks [18], [19] connecting multiple base stations). Note also that as reported in [18], an outdoor testbed in Berlin, Germany was successfully implemented to make such network coordination feasible, which consisted of four base station sites (seven sectors) connected via $1 \mathrm{~Gb} / \mathrm{s}$ optical-fibre and free space optical links. This example suggests that a network configuration with a CR network manager connected to SU-BS and PU-BS via optical fibre links is actually feasible in a practical cognitive radio network. Furthermore, it has been shown in various other works that some level of primary cooperation can benefit both the primary and secondary users [20], [21]. Finally, to financially justify PU-BS cooperation, the primary network service provider can also adopt a pricing system via which it can charge the CR network manager for providing CSI information. The CR network manager can recover this cost by charging each SU a nominal service fee. Once equipped with the real-time full CSI information, the CR network manager assigns the real-time channel realization $\left(g_{1}, g_{0}\right)$ to an optimal channel partition and the corresponding partition index is then sent to the SU-TX (and to the SU-RX for decoding purposes) via a delay-free and error-free [32] finite-rate feedback link. The SU-TX then uses the power codebook element associated with this index for data transmission. It was shown in [13] that without the presence of the CR network manager, and thus without the ability to jointly quantize the combined channel space, the SU capacity performance is significantly degraded if one carries out separate quantization of $g_{1}$ and $g_{0}$. Under such a network configuration, we derive various optimal and suboptimal quantized power allocation algorithms for secondary outage minimization. Motivated by limitations of existing practical backhaul links, we also consider the case where only quantized information of $g_{0}$ is available at the CR network manager. Finally, we also propose a sub-optimal quantized power allocation algorithm for the achievable sumrate outage minimization for a cognitive multiple-access channel with multiple secondary users and a single primary user.

The key contributions of this paper are listed as follow:

1) The difficulty of the outage minimization problem is due to the lack of an explicit expression for the outage probability with quantized power allocation (QPA). Thus one cannot directly exploit the Generalized Lloyd Algorithm (GLA) with a Lagrangian distortion, as we used in [13], 
to solve the outage minimization Problem with limited feedback. As an alternative, a sigmoid function can be used to approximate the indicator function [25], so as to obtain an approximate closed-form expression of outage probability. This approach then can be combined with GLA to design a suboptimal QPA algorithm, termed as the GLASFA method in this paper. We also investigate a general approach based on a simulation based optimization method called the 'Simultaneous Perturbation Stochastic Approximation algorithm (SPSA)' [23]. However, its high computational complexity coupled with a long convergence time limits its applicability. To overcome these drawbacks, we propose a novel lowcomplexity optimal QPA approach. To this end, we first prove that the optimal channel partition regions possess a "stepwise" structure, which helps us explicitly formulate the outage minimization problem, and then solve the corresponding optimization problem using the associated Karush-Kuhn-Tucker (KKT) necessary optimality conditions. The non-convexity of the problem however ensures that the solutions obtained are only locally optimum.

2) We also consider the situation when the CR network manager is assumed to be located at the SU-BS, and can obtain full information of $g_{1}$ without any backhaul link. In this case, the PU-BS simply has to cooperate with the SU-BS (or the CR network manager) by sending the information of $g_{0}$. Instead of assuming an optical fibre link to obtain full information of $g_{0}$ from PU$\mathrm{BS}$, we also consider the practical case of a finite rate feedback link (e.g. due to a microwave backhaul link) to obtain quantized information of $g_{0}$ from PU-BS. In this case, the CR network manager designs the optimal QPA algorithm based on full information of $g_{1}$ but quantized information of $g_{0}$ (we call it the QPA-Q $g_{0}$ algorithm).

3) As the number of feedback bits goes to infinity, we show that the power level for the last quantization region approaches zero, allowing us to derive a useful low-complexity suboptimal quantized power allocation algorithm called 'ZPiORA' for high rate quantization. Simulation results illustrate that 'ZPiORA' significantly outperforms the GLASFA method.

4) We also derive a number of significant useful properties related to the channel quantizer structure as the number of feedback bits approaches infinity: (a) under an active AIP constraint, the length of interval between any two adjacent quantization thresholds on $g_{0}$ axis is asymptotically the same, and (b) while when the AIP constraint is inactive, the ratio between any two adjacent quantization thresholds on $g_{1}$ axis asymptotically becomes identical. With these properties, we derive explicit expressions for asymptotic (as the number of feedback bits approaches infinity) behavior of the SU outage probability with quantized power allocation for high resolution quantization.

5) We then extend our SU outage minimization problem to the case of an achievable sum-rate outage minimization problem for multiple secondary users (SUs) forming a cognitive multiple-access channel (CMAC). It is seen that the quantization region structure is too complex for this case to be explicitly formulated. Hence we design a suboptimal quantized power allocation algorithm using the sigmoid function approximation to tackle the problem numerically.

6) Finally, we present a comprehensive set of numerical results which illustrate that with only 6 bits of feedback, the designed locally optimal QPA algorithms provide secondary outage probability very close to that achieved by full CSI. With 2-4 bits of feedback, ZPiORA provides a comparable performance, thus making it an attractive choice for large number of feedback bits case. Numerical studies also depict that ZPiORA performs better than two other suboptimal algorithms constructed using existing approximations in the literature. It is also shown that the derived asymptotic outage behavior approximates the true outage behaviour achieved by the locally optimal QPA extremely well as the number of feedback bits becomes large. Finally, numerical results regarding the sum-rate outage performance for the CMAC case with limited feedback are also provided.

This paper is organized as follows. Section II introduces the system model and the problem formulation based on the full CSI assumption. Section III presents the joint design of the optimal channel partition regions and an optimal power codebook algorithm. A low-complexity suboptimal quantized power allocation strategy is also derived using novel interesting properties of the quantizer structure and optimal quantized power codebooks. In Section IV, the asymptotic behavior of SU outage probability for high resolution quantization is investigated. A discussion on how to design a suboptimal QPA in the case of multiple SUs forming a CMAC is provided in Section V. Simulation results are given in Section VI, followed by concluding remarks in Section VII. Note that some of these results in this paper have been partially presented at a conferece [33]. This jounral version contains a rigorous and more substantial treatment of the secondary outage minimization problem in spectrum sharing and various other extensions such as considering multiple secondary users and the case of quantized $g_{0}$ information.

\section{System Model AND Problem Formulation}

We consider an infrastructure-based spectrum sharing network where a SU communication uplink to the SU-BS coexists with a PU link (to the PU-BS) within a narrowband channel. Regardless of the on/off status of PU, the SU is allowed to access the band which is originally allocated to PU, so long as the impact of the transmission of SU does not reduce the received signal quality of PU below a prescribed level. All channels here are assumed to be slow (appropriate for the outage probability as a performance metric) Rayleigh block fading channels. Let $g_{1}=\left|h_{1}\right|^{2}$ and $g_{0}=\left|h_{0}\right|^{2}$, denote the nonnegative real-valued instantaneous channel power gains for the links from SU-TX to SU-BS and SU-TX to PU-BS respectively (where $h_{1}$ and $h_{0}$ are corresponding complex zero-mean circularly symmetric channel amplitude gains). The exponentially distributed channel power gain $g_{1}$ and $g_{0}$, are statistically mutually independent and, without loss of 
generality (w.l.o.g), are assumed to have unity mean. It has been common practice to ignore the primary interference at the secondary receiver in many papers such as [5], [6], [13], whereas papers such as [14] consider primary interference explicitly assuming the primary transmitter (PU-TX) always transmits with a constant power, which along with the PUTX to SU-RX channel gain is known at the SU-BS. To avoid complications due to the added level of knowledge needed at the SU-BS in this framework, similar to [29], we consider any other interference from outside of the secondary network at the SU-BS, including the interference from the PU-TX, as additive Gaussian noise. This is based on the assumption that the primary uses a Gaussian codebook for transmission. The additive noises at SU-BS and PU-BS are assumed to be independent circular symmetric complex Gaussian (CSCG) random variables with, w.l.o.g, zero mean and unit variance.

Given a channel realization $\left(g_{0}, g_{1}\right)$, let the instantaneous transmit power (with full CSI) at the SU-TX be represented by $p\left(g_{0}, g_{1}\right)$, then the maximum mutual information of the SU for this narrowband spectrum sharing system can be expressed as $R\left(g_{1}, p\left(g_{0}, g_{1}\right)\right)=\frac{1}{2} \log \left(1+g_{1} p\left(g_{0}, g_{1}\right)\right)$, where $\log$ represents the natural logarithm. The outage probability of SU-TX with a pre-specified transmission rate $r_{0}$, is given as, $P_{\text {out }}=\operatorname{Pr}\left\{R\left(g_{1}, p\left(g_{0}, g_{1}\right)\right)<r_{0}\right\}$, where $\operatorname{Pr}\{A\}$ indicates the probability of event $A$ occurring. Using the interference temperature concept in [5], a common way to protect PU's received signal quality is by imposing either an average or a peak interference power (AIP/PIP) constraint at the PU-RX. In [22], it was demonstrated that the AIP constraint is more flexible and favorable than the PIP constraint in the context of transmission over fading channels. Let $Q_{a v}$ denotes the average interference power limit tolerated by PU-RX, then the AIP constraint can be written as, $E\left[g_{0} p\left(g_{0}, g_{1}\right)\right] \leq Q_{a v}$.

The following optimal power allocation problem that minimizes the outage probability of SU in a narrowband spectrum sharing with one PU, under both a long term average transmit power (ATP) constraint at SU-TX and an AIP constraint at the PU-RX, was considered in [4]

$$
\begin{array}{ll}
\underset{p\left(g_{0}, g_{1}\right) \geq 0}{\operatorname{minimize}} & \operatorname{Pr}\left\{\frac{1}{2} \log \left(1+g_{1} p\left(g_{0}, g_{1}\right)\right)<r_{0}\right\} \\
\text { subject to } & E\left[p\left(g_{0}, g_{1}\right)\right] \leq P_{a v}, \\
& E\left[g_{0} p\left(g_{0}, g_{1}\right)\right] \leq Q_{a v}
\end{array}
$$

where $P_{a v}$ is the maximum average transmit power at SU-TX.

With the assumption that perfect CSI of both $g_{0}$ and $g_{1}$ is available at the SU-TX, the optimal power allocation scheme for Problem (1) is given by [4]: $p^{*}\left(g_{0}, g_{1}\right)=$ $\left\{\begin{array}{lc}\frac{c}{g_{1}}, & \text { if } \lambda_{f}^{*}+\mu_{f}^{*} g_{0}<\frac{g_{1}}{c} \\ 0, & \text { otherwise }\end{array}\right.$, where $c=e^{2 r_{0}}-1$, and $\lambda_{f}^{*}, \mu_{f}^{*}$ are the optimal nonnegative Lagrange multipliers associated with the ATP constraint and the AIP constraint, respectively, which can be obtained by solving $\lambda_{f}^{*}\left(E\left[p\left(g_{0}, g_{1}\right)\right]-P_{a v}\right)=0$ and $\mu_{f}^{*}\left(E\left[g_{0} p\left(g_{0}, g_{1}\right)\right]-Q_{a v}\right)=0$.

However, the assumption of full CSI at the SU-TX (especially that of $g_{0}$ ) is usually unrealistic and difficult to implement in practical systems, especially when this channel is not time-division duplex (TDD). In the next section, we are therefore interested in designing a power allocation strategy

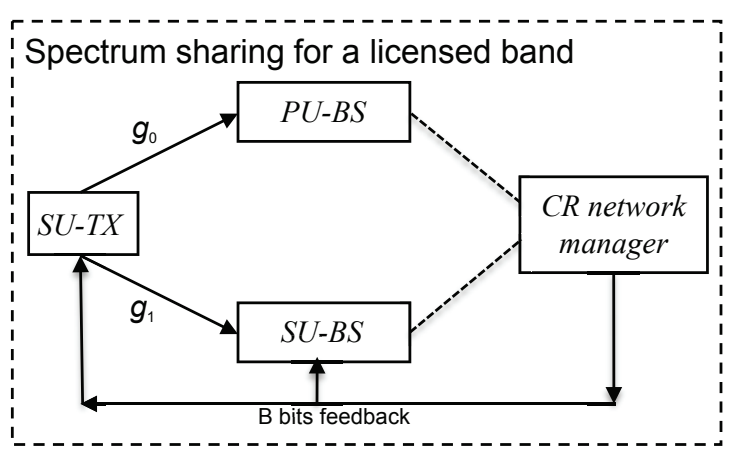

Fig. 1. System model for narrowband spectrum sharing scenario with limited rate feedback.

of the outage probability minimization Problem (1) based on quantized CSI at the SU-TX acquired via a no-delay and errorfree ${ }^{1}$ feedback link with limited rate.

\section{Optimum QuANTIZED Power Allocation (QPA) WITH IMPERFECT $g_{1}$ AND $g_{0}$ AT SU-TX}

\section{A. Optimal QPA with limited rate feedback strategy}

As shown in Fig.1, following our earlier work [13], we assume that there is a central controller termed as CR network manager who can obtain perfect information of $g_{0}$ and $g_{1}$, from PU-RX at the PU base station and SU-RX at the SU base station respectively, possibly over fibre-optic links, and then forward some appropriately quantized $\left(g_{0}, g_{1}\right)$ information to SU-TX through a finite-rate feedback link. For further details on the justification of resulting benefits due this assumption, see [13]. Under such a network modelling assumption, given B bits of feedback, a power codebook $\mathcal{P}=\left\{p_{1}, \ldots, p_{L}\right\}$ of cardinality $L=2^{B}$, is designed offline purely on the basis of the statistics of $g_{0}$ and $g_{1}$ information at the CR network manager. This codebook is also known a priori by both SU-TX and SU-RX for decoding purposes. Given a channel realization $\left(g_{0}, g_{1}\right)$, the $\mathrm{CR}$ network manager employs a deterministic mapping from the current instantaneous $\left(g_{0}, g_{1}\right)$ information to one of $L$ integer indices (let $\mathcal{I}\left(g_{0}, g_{1}\right)$ denote the mapping, which partitions the vector space of $\left(g_{0}, g_{1}\right)$ into $L$ regions $\mathcal{R}_{1}, \ldots, \mathcal{R}_{L}$, defined as $\mathcal{I}\left(g_{0}, g_{1}\right)=j$, if $\left(g_{0}, g_{1}\right) \in$ $\left.\mathcal{R}_{j}, \quad j=1, \ldots, L\right)$, and then sends the corresponding index $j=\mathcal{I}\left(g_{0}, g_{1}\right)$ to the SU-TX (and the SU-RX) via the feedback link. The SU-TX then uses the associated power codebook element (e.g., if the feedback signal is $j$, then $p_{j}$ will be used as the transmission power) to adapt its transmission strategy.

Remark 1: Note that the CR network manager could be assumed to be located at the SU-BS for the current setup and in this case, the PU-BS simply has to cooperate with the SU-BS by sending the real-time full CSI information of $g_{0}$. However, for future generalization of our work to a multi-cell cognitive network scenario, we assume that the CR network

\footnotetext{
${ }^{1}$ Since we consider a slow block-fading environment and only a few bits of CSI feedback (per fading block) are required, the feedback delay is significantly less than the coherence time of the fading channels concerned. Thus, according to [32], the effect of feedback delay can be negligible. Moreover, with such small data rate, error-free feedback can be easily achieved by employing efficient and strong error control coding over the feedback link [32].
} 
manager is a separate entity, which can obtain information from multiple PU-BS and SU-BS if necessary.

Define an indicator function $X_{j}, j=1, \ldots, L$, as $X_{j}=$ $\left\{\begin{array}{ccc}1, & \text { if } & \frac{1}{2} \log \left(1+g_{1} p_{j}\right)<r_{0} \\ 0, & \text { otherwise } & \text {. Let } \operatorname{Pr}\left(\mathcal{R}_{j}\right), E\left[\bullet \mid \mathcal{R}_{j}\right] \text { rep- }\end{array}\right.$ resent $\operatorname{Pr}\left(\left(g_{0}, g_{1}\right) \in \mathcal{R}_{j}\right)$ and $E\left[\bullet \mid\left(g_{0}, g_{1}\right) \in \mathcal{R}_{j}\right]$, respectively. Then the SU outage probability minimization problem with limited feedback can be formulated as

$$
\begin{array}{ll}
\underset{p_{j} \geq 0, \mathcal{R}_{j} \forall j}{\operatorname{minimize}} & \sum_{j=1}^{L} E\left[X_{j} \mid \mathcal{R}_{j}\right] \operatorname{Pr}\left(\mathcal{R}_{j}\right) \\
\text { subject to } & \sum_{j=1}^{L} E\left[p_{j} \mid \mathcal{R}_{j}\right] \operatorname{Pr}\left(\mathcal{R}_{j}\right) \leq P_{a v}, \\
& \sum_{j=1}^{L} E\left[g_{0} p_{j} \mid \mathcal{R}_{j}\right] \operatorname{Pr}\left(\mathcal{R}_{j}\right) \leq Q_{a v} .
\end{array}
$$

Thus the key problem to solve here is the joint optimization of the channel partition regions and the power codebook such that the outage probability of SU is minimized under the above constraints.

The dual problem of (2) is expressed as, maximize $\lambda \geq 0, \mu \geq 0$ $g(\lambda, \mu)-\lambda P_{a v}-\mu Q_{a v}$, where $\lambda, \mu$ are the nonnegative Lagrange multipliers associated with the ATP and AIP constraints in Problem (2), and the Lagrange dual function $g(\lambda, \mu)$ is defined as

$g(\lambda, \mu)=\underset{p_{j} \geq 0, \mathcal{R}_{j}, \forall j}{\operatorname{minimize}} \sum_{j=1}^{L} E\left[X_{j}+\left(\lambda+\mu g_{0}\right) p_{j} \mid \mathcal{R}_{j}\right] \operatorname{Pr}\left(\mathcal{R}_{j}\right)$.

The procedure we use to solve the above dual problem is:

Step 1: With fixed values of $\lambda$ and $\mu$, find the optimal solution (power codebook and quantization regions) for the Lagrange dual function (3).

Step 2: Find the optimal $\lambda$ and $\mu$ by solving the dual problem using subgradient search method, i.e, updating $\lambda, \mu$ until convergence using

$$
\begin{aligned}
& \lambda^{l+1}=\left[\lambda^{l}-\alpha^{l}\left(P_{a v}-\sum_{j=1}^{L} E\left[p_{j} \mid \mathcal{R}_{j}\right] \operatorname{Pr}\left(\mathcal{R}_{j}\right)\right)\right]^{+}, \\
& \mu^{l+1}=\left[\mu^{l}-\beta^{l}\left(Q_{a v}-\sum_{j=1}^{L} E\left[g_{0} p_{j} \mid \mathcal{R}_{j}\right] \operatorname{Pr}\left(\mathcal{R}_{j}\right)\right)\right]^{+},
\end{aligned}
$$

where $l$ is the iteration number, $\alpha^{l}$, $\beta^{l}$ are positive scalar step sizes for the $l$-th iteration satisfying $\sum_{l=1}^{\infty} \alpha^{l}=$ $\infty, \sum_{l=1}^{\infty}\left(\alpha^{l}\right)^{2}<\infty$ and similarly for $\beta^{l}$, and $[x]^{+}=$ $\max (x, 0)$.

Remark 2: A general method to solve Step 1 is to employ a simulation-based optimization algorithm called Simultaneous Perturbation Stochastic Approximation (SPSA) algorithm (for a step-by-step guide to implementation of SPSA, see [23]), where one can use the objective function of Problem (3) as the loss function and the optimal power codebook elements for each channel partition are obtained via a randomized stochastic gradient search technique. Note that due to the presence of the indicator function and no explicit expression being available for the outage probability with quantized power allocation, we can't directly exploit the Generalized Lloyd Algorithm (GLA) with a Lagrangian distortion, as we used in [13], to solve Problem (3). SPSA uses a simulationbased method to compute the loss function and then estimates the gradient from a number of loss function values computed by randomly perturbing the power codebook. Note that SPSA results in a local minimum (similar to GLA), but is computationally highly complex and the convergence time is also quite long.

Due to the high computational complexity of SPSA and its long convergence time to solve Problem (3), we will next derive a low-complexity approach for solving Problem (3). However, due to the original problem (2) not being convex with respect to the power codebook elements, the optimal solution we can obtain is also locally optimal.

Let $\mathcal{P}=\left\{p_{1}, \ldots, p_{L}\right\}$, where $p_{1}>\cdots>p_{L} \geq 0$, and the corresponding channel partitioning $\mathcal{R}_{1}, \ldots, \mathcal{R}_{L}$ denote an optimal solution to Problem (3). Let $p\left(\mathcal{I}\left(g_{0}, g_{1}\right)\right)$ represent the mapping from instantaneous $\left(g_{0}, g_{1}\right)$ information to the allocated power level. We can then obtain the following result:

Lemma 1: Let $\left\{v_{1}, \ldots, v_{L}\right\}$ denote the optimum quantization thresholds on the $g_{1}$ axis $\left(0<v_{1}<\cdots<\right.$ $\left.v_{L}\right)$ and $\left\{s_{1}, \ldots, s_{L-1}\right\}$ indicate the optimum quantization thresholds on the $g_{0}$ axis $\left(0<s_{1}<\cdots<s_{L-1}\right)$. Then we have $\forall j, j=1, \ldots, L-1, p\left(\mathcal{I}\left(g_{0}, g_{1}\right)\right)=$

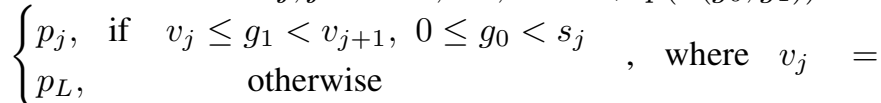
$\frac{c}{p_{j}}, j=1, \ldots, L$, and for $\forall j, j=1, \ldots, L-1$, when $\mu>0, s_{j}=\frac{1}{\mu\left(p_{j}-p_{L}\right)}-\frac{\lambda}{\mu}$, while when $\mu=0, s_{j}=\infty$, then condition $0 \leq g_{0}<s_{j}$ boils down to $\lambda<\frac{1}{p_{j}-p_{L}}$. The region $\mathcal{R}_{L}$ includes two parts : the set $\mathcal{R}_{L 1}=\left\{\left(g_{0}, g_{1}\right): v_{j} \leq\right.$ $\left.g_{1}<v_{j+1}, g_{0} \geq s_{j}, \forall j=0, \ldots, L-1\right\}$ with $s_{0}=0, v_{0}=0$ and the set $\mathcal{R}_{L 2}=\left\{\left(g_{0}, g_{1}\right): g_{1} \geq v_{L}, g_{0} \geq 0\right\}$. The entire set $\mathcal{R}_{L 1}$ is in outage.

Proof: See Appendix A.

Remark 3: When $\mu>0$, which implies that the AIP constraint is active, from Lemma 1 , the optimum partition regions possess a stepwise structure, as shown in Fig.2. When $\mu=0$, i.e, the AIP constraint is inactive and only ATP constraint is active (we must have $\lambda>0$ ), Problem (2) becomes a scalar quantization problem involving quantizing $g_{1}$ only, and Lemma 1 reduces to : $p\left(\mathcal{I}\left(g_{1}\right)\right)=$ $\left\{\begin{array}{lc}p_{j}, & \text { if } \quad v_{j} \leq g_{1}<v_{j+1}, \forall j, j=1, \ldots, L-1, \quad \text { where } \\ p_{L}, & \text { otherwise }\end{array}\right.$ $\lambda<\frac{1}{p_{j}-p_{L}}, \forall j=1,2, \ldots, L-1$ and the two subregions of $\mathcal{R}_{L}$ become $\mathcal{R}_{L 1}=\left\{g_{1}: 0 \leq g_{1}<v_{1}\right\}$ and $\mathcal{R}_{L 2}=\left\{g_{1}: g_{1} \geq v_{L}\right\}$, and $\mathcal{R}_{L 1}$ is in outage. Note that in this case we must have $Q_{a v} \geq P_{a v}$, due to $Q_{a v} \geq$ $\sum_{j=1}^{L} E\left[g_{0} p_{j} \mid \mathcal{R}_{j}\right] \operatorname{Pr}\left(\mathcal{R}_{j}\right)=\sum_{j=1}^{L} E\left[p_{j} \mid \mathcal{R}_{j}\right] \operatorname{Pr}\left(\mathcal{R}_{j}\right)=P_{a v}$, where the last equality follows from the fact the $E\left[g_{0} \mid \mathcal{R}_{j}\right]=E\left[g_{0}\right]=1$ since $\mathcal{R}_{j}$ is formed purely based on the values of $g_{1}$, which is independent of $g_{0}$. Note also that one can easily prove the converse, that when $Q_{a v} \geq P_{a v}$, one must have $\mu=0$.

From Lemma 1, (due to the fading channels being independently exponentially distributed with unity mean) Problem (2) becomes, 


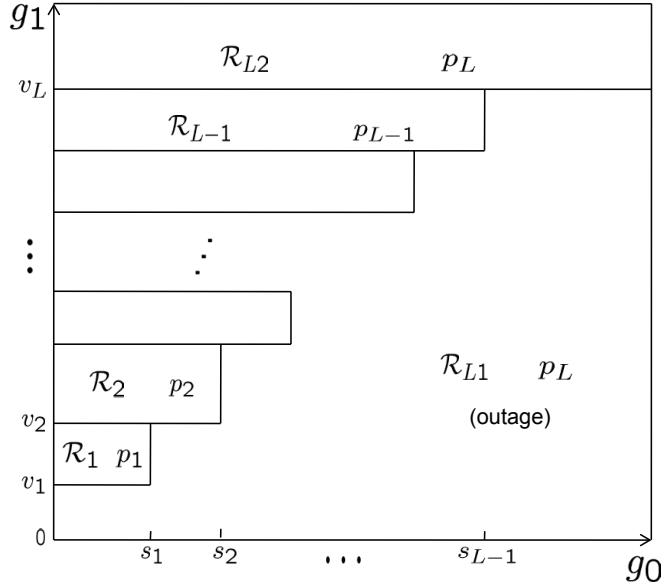

Fig. 2. The stepwise structure of optimum quantization regions for $\mu>0$ case.

$\underset{p_{j} \geq 0, \forall j}{\operatorname{minimize}} \quad P_{\text {out }}^{L}=1-e^{-v_{1}}+\sum_{j=1}^{L-1}\left(e^{-v_{j}}-e^{-v_{j+1}}\right) e^{-s_{j}}$

subject to

$p_{L}+\sum_{j=1}^{L-1}\left(p_{j}-p_{L}\right)\left(e^{-v_{j}}-e^{-v_{j+1}}\right)\left(1-e^{-s_{j}}\right) \leq P_{a v}$

$p_{L}+\sum_{j=1}^{L-1}\left(p_{j}-p_{L}\right)\left(e^{-v_{j}}-e^{-v_{j+1}}\right)\left(1-e^{-s_{j}}\left(1+s_{j}\right)\right) \leq Q_{a v}$,

where $P_{\text {out }}^{L}$ denotes the outage probability with $B=\log _{2} L$ bits feedback QPA, $v_{j}=\frac{c}{p_{j}}, j=1, \ldots, L$ and for $\forall j, j=$ $1, \ldots, L-1$, when $\mu>0, s_{j}=\frac{1}{\mu\left(p_{j}-p_{L}\right)}-\frac{\lambda}{\mu}$, whereas when $\mu=0, s_{j}=\infty$. Although the above optimization problem may be verified to be non-convex, we can employ the KKT necessary conditions to find a local minimum for Problem (5). Taking the partial derivative of first order of the Lagrangian of Problem (5) over $p_{j}, j=1, \ldots, L-1$, and setting it to zero, we can obtain

$$
\begin{aligned}
& \left(e^{-v_{j}}-e^{-v_{j+1}}\right)\left[\lambda\left(1-e^{-s_{j}}\right)+\mu\left(1-e^{-s_{j}}\left(1+s_{j}\right)\right)\right] \\
& =e^{-v_{j}} \frac{c}{p_{j}^{2}}\left[\hat{f}\left(p_{j-1}\right)-\hat{f}\left(p_{j}\right)\right], \quad 1 \leq j \leq L-1 ;
\end{aligned}
$$

where $\hat{f}\left(p_{0}\right)=1$ and $\hat{f}\left(p_{j}\right)=\left(p_{j}-p_{L}\right)\left(\lambda+\mu\left(1-e^{-s_{j}}\right)\right), 1 \leq$ $j \leq L-1$. (6) also can be rewritten as $j=1, \ldots, L-1$,

$$
p_{j+1}=\frac{c}{v_{j}-\ln \left(1-\frac{\frac{c}{p_{j}^{2}}\left[\hat{f}\left(p_{j-1}\right)-\hat{f}\left(p_{j}\right)\right]}{\lambda\left(1-e^{-s_{j}}\right)+\mu\left(1-e^{-s_{j}}\left(1+s_{j}\right)\right)}\right)},
$$

Equating the partial derivative of the Lagrangian function of Problem (5) over $p_{L}$ to zero gives,

$$
\begin{aligned}
& \sum_{j=1}^{L-1}\left(e^{-v_{j}}-e^{-v_{j+1}}\right)\left[\lambda\left(1-e^{-s_{j}}\right)+\mu\left(1-e^{-s_{j}}\left(1+s_{j}\right)\right)\right] \\
& +e^{-v_{L}} \frac{c}{p_{L}^{2}} \hat{f}\left(p_{L-1}\right)=\lambda+\mu .
\end{aligned}
$$

Optimal values of $\lambda$ and $\mu$ can be determined by solving

$$
\begin{aligned}
& \lambda\left[p_{L}+\sum_{j=1}^{L-1}\left(p_{j}-p_{L}\right)\left(e^{-v_{j}}-e^{-v_{j+1}}\right)\left(1-e^{-s_{j}}\right)-P_{a v}\right]=0 \\
& \mu\left[p_{L}+\sum_{j=1}^{L-1}\left(p_{j}-p_{L}\right)\left(e^{-v_{j}}-e^{-v_{j+1}}\right)\left(1-e^{-s_{j}}\left(1+s_{j}\right)\right)\right. \\
& \left.\quad-Q_{a v}\right]=0 .
\end{aligned}
$$

Thus, for fixed values $\lambda$ and $\mu$, we need to solve the $L$ equations given by (7), (8) to obtain the power codebook. Given $p_{1}$ and $p_{L}$, from (7) we can successively compute $p_{2}, \ldots, p_{L-1}$, and then we can jointly solve the equation (7) with $j=L-1$ and equation (8) numerically for $p_{1}$ and $p_{L}$. The optimal value of $\lambda$ and $\mu$ can be obtained by solving (9) with a subgradient method, i,e. by updating $\lambda$ and $\mu$ until convergence using (4). One can thus repeat the above two steps (i.e, given $\lambda$ and $\mu$ find the optimal power levels, and then using the resulting optimal power levels update $\lambda$ and $\mu$ ) iteratively until a satisfactory convergence criterion is met. This procedure can be formally summarized as:

a) First, if $P_{a v} \leq Q_{a v}$, we must have $\mu=0, \lambda>0$. Starting with an arbitrary positive initial value for $\lambda$, solve (6), (8) to obtain a power codebook $\left\{p_{1}, \ldots, p_{L}\right\}$, and then use this codebook to update $\lambda$ by (4). Repeat these steps until convergence and the final codebook will be an optimal power codebook for Problem (5).

b) If $P_{a v}>Q_{a v}$, we must have $\mu>0$ by contradiction (since if $\mu=0$, we must have $P_{a v} \leq Q_{a v}$ ). Let $\lambda=$ 0 , then solving KKT conditions gives an optimal value of $\mu$ and corresponding power codebook $\left\{p_{1}, \ldots, p_{L}\right\}$. With this codebook, if $\sum_{j=1}^{L} E\left[p_{j} \mid \mathcal{R}_{j}\right] \operatorname{Pr}\left(\mathcal{R}_{j}\right) \leq P_{a v}$, then it is an optimal power codebook for Problem (5). Otherwise we must have $\lambda>0$ too, in which case, starting with arbitrary positive initial values for $\lambda$ and $\mu$, obtain the corresponding power codebook $\left\{p_{1}, \ldots, p_{L}\right\}$, and then update $\lambda$ and $\mu$ by (4). Repeat these steps until convergence and the final codebook will be an optimal power codebook for Problem (5).

\section{B. QPA algorithm based on quantized $g_{0}$ from $P U-B S$ and full $g_{1}$ from $S U-B S$}

In this section (see Remark 1), we assume the CR network manager is located at the SU-BS and thus is able to obtain full information on $g_{1}$ without any backhaul link. In this case, the PU-BS simply has to cooperate with the SU-BS (or equivalently, the CR network manager) by sending CSI of $g_{0}$. Motivated by finite-rate constraints of existing wireless backhaul links, here we consider using a finite rate feedback link to obtain quantized information of $g_{0}$ from PU-BS. In this case, the CR network manager designs the optimal QPA algorithm based on full information of $g_{1}$ but quantized information of $g_{0}$ (we call it the QPA-Q $g_{0}$ algorithm).

More specifically, PU-BS quantizes $g_{0}$ by minimizing its corresponding squared distortion measure $\sum_{n=1}^{L^{\prime}} E\left[\left(g_{0}-\right.\right.$ $\left.\left.g_{0 n}^{\prime}\right)^{2} \mid R_{n}\right] \operatorname{Pr}\left(R_{n}\right)$ using the typical Lloyd Algorithm, where $g_{0 n}^{\prime}$ is the reconstruction vector for $g_{0}$ in the quantization 
region $R_{n}$. Then PU-BS maps the instantaneous $g_{0}$ information into one of $L^{\prime}$ integer indices and sends the index to the CR network manager. Since the codebook of the PU-BS for quantizing $g_{0}$ is designed offline, the CR network manager can be made aware of this codebook a priori. The CR network manager then designs a locally optimal QPA-Q $g_{0}$ algorithm power codebook $\mathcal{P}=\left\{p_{1}, \ldots, p_{L}\right\}$ based on quantized $g_{0}$ (i.e., based on fixed quantization thresholds on the $g_{0}$ axis) and full $\mathbf{g}_{1}$ information, it then maps the received $g_{0}$ index together with instantaneous $g_{1}$ information into one of $L$ integer indices and sends the index to the SU-TX.

The key issue here is how to design the optimal QPA-Q $g_{0}$ algorithm. For simplicity, we assume $L=L^{\prime}$, although this method can be generalized to any $L^{\prime}$. With a set of given quantization thresholds on the $g_{0}$ axis, $0<s_{1}<\cdots<s_{L-1}$, let $\left\{v_{1}, \ldots, v_{L}\right\}$ denote the optimum quantization thresholds on the $g_{1}$ axis $\left(0<v_{1}<\cdots<v_{L}\right)$. Then, when $P_{a v} \leq Q_{a v}$, i.e., $\mu=0$, the AIP constraint is inactive, no matter what the index for quantized $g_{0}$ is, the QPA-Q $g_{0}$ algorithm is identical to the optimal QPA algorithm. When $P_{a v}>Q_{a v}$, i.e., $\mu>0$, the AIP constraint is active. In this case, using a proof similar to that of Lemma 1, we can show that the optimal quantization regions of QPA-Q $g_{0}$ has a stepwise structure as given in Lemma 1 but with known $g_{0}$ quantization thresholds. Then similar to (5), the outage probability minimization problem with limited feedback for QPA-Q $g_{0}$ case can be formulated as

$$
\begin{aligned}
& \underset{p_{L} \geq 0}{\operatorname{minimize}} P_{\text {out }}^{L}=1-e^{-v_{1}}+\sum_{j=1}^{L-1}\left(e^{-v_{j}}-e^{-v_{j+1}}\right) e^{-s_{j}} \\
& \text { subject to } \\
& p_{L}+\sum_{j=1}^{L-1} \frac{1}{\lambda+\mu s_{j}}\left(e^{-v_{j}}-e^{-v_{j+1}}\right)\left(1-e^{-s_{j}}\right) \leq P_{a v} \\
& p_{L}+\sum_{j=1}^{L-1} \frac{1}{\lambda+\mu s_{j}}\left(e^{-v_{j}}-e^{-v_{j+1}}\right)\left(1-e^{-s_{j}}\left(1+s_{j}\right)\right) \leq Q_{a v}, \\
& v_{j}=\frac{c}{p_{L}+\frac{1}{\lambda+\mu s_{j}}} \quad \forall j=1, \ldots, L, s_{L}=\infty
\end{aligned}
$$

The above problem first needs to be solved for $p_{L}$ by any appropriate nonlinear equation solver. After obtaining $p_{L}$, $p_{j}=p_{L}+\frac{1}{\lambda+\mu s_{j}}, \forall j=1, \ldots, L-1$, can be obtained easily. Numerical results illustrate that the outage performance gap between the QPA-Q $g_{0}$ algorithm and original QPA algorithm is considerably small especially for small $Q_{a v}$ values. See section VI for more details on simulation results .

\section{Suboptimal QPA Algorithm}

In this section, we derive a computationally efficient but suboptimal algorithm for the case when the number of feedback bits becomes large. When the number of feedback bits $B$ (or alternatively, $L$ ) goes to infinity, we can obtain the following Lemma that allows us to obtain this suboptimal but computationally efficient quantized power allocation algorithm for large but finite $L$.

Lemma 2: $\lim _{L \rightarrow \infty} p_{L}=0$

Proof: See appendix B.

Remark 4: Lemma 2 shows that regardless of whether $\mu>0$ or $\mu=0$, with high rate quantization, the power level for the last region $\mathcal{R}_{\mathcal{L}}$ approaches zero, which also implies the following as $L \rightarrow \infty$ :

1) The non-outage part of $\mathcal{R}_{\mathcal{L}}$, given by $\mathcal{R}_{\mathcal{L}_{2}}$, disappears gradually. In other words, $\mathcal{R}_{\mathcal{L}} \rightarrow \mathcal{R}_{\mathcal{L} 1}$. Thus, when $L \rightarrow \infty$, $\mathcal{R}_{\mathcal{L}}$ becomes the outage region with zero power assigned to it.

2) When $\mu>0$, the quantization thresholds on the $g_{0}$ axis $s_{j} \rightarrow s_{j}^{\prime}$ (where $s_{j}^{\prime}=\frac{1}{\mu p_{j}}-\frac{\lambda}{\mu}$ ), which gives $v_{j}=c \lambda+c \mu s_{j}^{\prime}$, and it means all the points given by coordinates $\left(s_{j}^{\prime}, v_{j}\right)$ lie on the line of $g_{1}=c \lambda+c \mu g_{0}$. Therefore, as $L \rightarrow \infty$, the stepwise shape of the structure in $\mu>0$ case (i.e, the boundary between non-outage and outage regions) approaches the straight line $g_{1}=c \lambda+c \mu g_{0}$, which is consistent with the full CSI-based power allocation result in [4].

Thus, when $L$ is large, applying Lemma 2 (i.e, $p_{L} \rightarrow 0$ ) to Problem (5), the above $L$ KKT conditions (6) and (8) can be simplified into $L-1$ equations:

$$
\begin{gathered}
\left(e^{-v_{j}}-e^{-v_{j+1}}\right)\left[\lambda\left(1-e^{-s_{j}^{\prime}}\right)+\mu\left(1-e^{-s_{j}^{\prime}}\left(1+s_{j}^{\prime}\right)\right)\right] \\
=e^{-v_{j}} \frac{c}{p_{j}^{2}}\left[p_{j-1}\left(\lambda+\mu\left(1-e^{-s_{j-1}^{\prime}}\right)\right)-p_{j}\left(\lambda+\mu\left(1-e^{-s_{j}^{\prime}}\right)\right)\right], \\
\forall j=1, \ldots, L-1
\end{gathered}
$$

where when $\mu>0$, the quantization thresholds on the $g_{0}$ axis are given by $s_{j}^{\prime}=\frac{1}{\mu p_{j}}-\frac{\lambda}{\mu}, s_{0}^{\prime}=0$, and $p_{0}=\frac{1}{\lambda+\mu s_{0}^{\prime}}$, while when $\mu=0, s_{j}^{\prime}=\infty, s_{0}^{\prime}=0$, and $p_{0}=\frac{1}{\lambda}$. (11) can be also written as (12). Thus, for given values of $\lambda$ and $\mu$, starting with a specific value of $p_{1}$, we can successively compute $p_{2}, \ldots, p_{L-1}$ using the first equation of (12) (recall that $v_{j}=\frac{c}{p_{j}}$ ). Then the second equation in (12) becomes an equation in $p_{1}$ only, which can be solved easily using a suitable nonlinear equation solver. We call this suboptimal QPA algorithm as 'Zero Power in Outage Region Approximation'(ZPiORA), which is applicable to the case of a large number of feedback bits, where the exact definition of "large" will be dependent on the system parameters. Through simulation studies, we will illustrate that for our choice of system parameters, ZPiORA performs well even for as low as $B=2$ bits of feedback.

Alternative suboptimal algorithms: For comparison purposes, we also propose two alternative suboptimal algorithms described below:

(1) The first suboptimal algorithm is based on an equal average power per (quantized) region (EPPR) approximation algorithm, proposed in [24] in a non-cognitive or typical primary network setting for an outage minimization problem with only an ATP constraint. More specifically, by applying the mean value theorem (similar to [24]) into the KKT conditions (6) with $j=2, \ldots, L-1$, we can easily obtain that $p_{j}\left(e^{-v_{j}}-e^{-v_{j+1}}\right)[\lambda(1-$ $\left.\left.e^{-s_{j}}\right)+\mu\left(1-e^{-s_{j}}\left(1+s_{j}\right)\right)\right] \approx p_{j-1}\left(e^{-v_{j-1}}-\right.$ $\left.e^{-v_{j}}\right)\left[\lambda\left(1-e^{-s_{j-1}}\right)+\mu\left(1-e^{-s_{j-1}}\left(1+s_{j-1}\right)\right)\right], \quad j=$ $2, \ldots, L-2$. Adding the two equations of (9) together and applying (8), we have $\sum_{j=1}^{L-1} p_{j}\left(e^{-v_{j}}-\right.$ $\left.e^{-v_{j+1}}\right)\left[\lambda\left(1-e^{-s_{j}}\right)+\mu\left(1-e^{-s_{j}}\left(1+s_{j}\right)\right)\right]=\lambda P_{a v}+$ $\mu Q_{a v}-e^{-v_{L}} \frac{c}{p_{L}}\left(p_{L-1}-p_{L}\right)\left(\lambda+\mu\left(1-e^{-s_{L-1}}\right)\right)$. Since $e^{-v_{L}} \frac{c}{p_{L}}\left(p_{L-1}-p_{L}\right)\left(\lambda+\mu\left(1-e^{-s_{L-1}}\right)\right)$ can be approximated as $p_{L-1}\left(e^{-v_{L-1}}-e^{-v_{L}}\right)\left[\lambda\left(1-e^{-s_{L-1}}\right)+\right.$ 


$$
\begin{gathered}
p_{j+1}=\frac{c}{v_{j}-\ln \left(1-\frac{\frac{c}{p_{j}^{2}}\left[p_{j-1}\left(\lambda+\mu\left(1-e^{-s_{j-1}^{\prime}}\right)\right)-p_{j}\left(\lambda+\mu\left(1-e^{-s_{j}^{\prime}}\right)\right)\right]}{\lambda\left(1-e^{-s_{j}^{\prime}}\right)+\mu\left(1-e^{-s_{j}^{\prime}}\left(1+s_{j}^{\prime}\right)\right)}\right)} \\
\forall j=1, \ldots, L-2 ; \\
\frac{c\left(1-e^{-s_{L-1}^{\prime}}\right)+\mu\left(1-e^{-s_{L-1}^{\prime}}\left(1+s_{L-1}^{\prime}\right)\right)}{\frac{c}{p_{L-1}^{2}}\left[p_{L-2}\left(\lambda+\mu\left(1-e^{-s_{L-2}^{\prime}}\right)\right)-p_{L-1}\left(\lambda+\mu\left(1-e^{-s_{L-1}^{\prime}}\right)\right)\right]}=1 .
\end{gathered}
$$

$\left.\mu\left(1-e^{-s_{L-1}}\left(1+s_{L-1}\right)\right)\right]$ by using the mean value theorem, we can obtain the following $L$ (approximate) equations, namely $p_{j}\left(e^{-v_{j}}-e^{-v_{j+1}}\right)\left[\lambda\left(1-e^{-s_{j}}\right)+\mu(1-\right.$ $\left.\left.e^{-s_{j}}\left(1+s_{j}\right)\right)\right] \approx \frac{\lambda P_{a v}+\mu Q_{a v}}{L}, j=1, \ldots, L-1$ and $p_{L}\left(1-\sum_{j=1}^{L-1}\left(e^{-v_{j}}-e^{-v_{j+1}}\right)\left[\lambda\left(1-e^{-s_{j}}\right)+\mu(1-\right.\right.$ $\left.\left.\left.e^{-s_{j}}\left(1+s_{j}\right)\right)\right]\right) \approx \frac{\lambda P_{a v}+\mu Q_{a v}}{L}$. Then one can jointly solve the above $L$ equations and two other equations ((6) with $j=1$ and (8)) for $\lambda, \mu, p_{j}, \forall j=1, \ldots, L$. We call this suboptimal algorithm as the "Modified EPPR (MEPPR)" approximation algorithm. Obviously, ZPiORA is computationally much simpler than this method, especially when $\mu>0$. Furthermore, from simulations, when $P_{a v}$ or $Q_{a v}$ is small, the performance of ZPiORA is always better than MEPPR. It is seen however that when both $P_{a v}$ and $Q_{a v}$ are large, for a small number of feedback bits, MEPPR may outperform ZPiORA, whereas with a sufficiently large number of feedback bits, ZPiORA is a more accurate approximation due to Lemma 2 (when $L$ is large, $p_{L}$ approaches zero, whereas MEPPR has $\left.p_{L}>0 \forall L\right)$. See Section V for more details. Note that, an equal probability per region (excluding the outage region) approximation algorithm employed in [10] for scalar quantization can not be applied to our case (vector quantization), since it will increase the computational complexity even further.

(2) The second algorithm is based on GLA with a sigmoid function approximation (GLASFA) method proposed by [25], where the sigmoid function is used to approximate the indicator function in the Lagrange dual function (3). More specifically, given a random initial power codebook, we use the nearest neighbor condition of Lloyd's algorithm with a Lagrangian distortion $d\left(\left(g_{0}, g_{1}\right), j\right)=X_{j}+\left(\lambda+\mu g_{0}\right) p_{j}$ to generate the optimal partition regions [26] given by, $\mathcal{R}_{j}=\left\{\left(g_{0}, g_{1}\right)\right.$ : $\left.X_{j}+\left(\lambda+\mu g_{0}\right) p_{j} \leq X_{i}+\left(\lambda+\mu g_{0}\right) p_{i}, \forall i \neq j\right\}$, $i, j=1, \ldots, L$. We then use the resulting optimal partition regions to update the power codebook by $p_{j} \approx \operatorname{argmin}_{p_{j} \geq 0} E\left[\sigma\left(k\left(\frac{1}{2} \log \left(1+g_{1} p_{j}\right)-r_{0}\right)\right)+(\lambda+\right.$ $\left.\left.\mu g_{0}\right)_{j} \mid R_{j}\right] \operatorname{Pr}\left(R_{j}\right)$ for $j=1, \ldots, L$, where we use the approximation $X_{j} \approx \sigma\left(k\left(\frac{1}{2} \log \left(1+g_{1} p_{j}\right)-r_{0}\right)\right)$, $\sigma(x)=\frac{1}{1+e^{x}}$ being the sigmoid function where the coefficient $k$ controls the sharpness of the approximation (for detailed guidelines on choosing $k$ see [25]). The above two steps of GLA are repeated until convergence. Numerical results illustrate that ZPiORA significantly outperforms this suboptimal method. See Section V for more details.

\section{AsyMPTOTIC OUTAGE BEHAVIOUR OF QPA UNDER High RESOLUTION QUANTIZATION}

In this section, we derive a number of asymptotic expressions for the SU outage probability when the number of feedback bits approaches infinity. To this end, we first derive some useful properties regarding the quantizer structure at high rate quantization:

Lemma 3: As the number of quantization regions $L \rightarrow \infty$, we can obtain the following result: with $\mu>0$, the optimum quantization thresholds on the $g_{0}$ axis satisfy $s_{1}^{\prime}-s_{0}^{\prime} \approx s_{2}^{\prime}-$ $s_{1}^{\prime} \approx \ldots \approx s_{L-1}^{\prime}-s_{L-2}^{\prime}$, where $s_{j}^{\prime}=\frac{1}{\mu p_{j}}-\frac{\lambda}{\mu}, j=1, \ldots, L-1$ and $s_{0}^{\prime}=0$. With $\mu=0$, the optimum quantization thresholds on the $g_{1}$ axis satisfy $\frac{v_{1}}{v_{0}} \approx \frac{v_{2}}{v_{1}} \cdots \approx \frac{v_{L-1}}{v_{L-2}}$, where $v_{j}=\frac{c}{p_{j}}, j=$ $1, \ldots, L-1$ and here $v_{0}=c \lambda$.

Proof: See Appendix C.

Lemma 4: In the high rate quantization regime, as $L \rightarrow \infty$, we have

$$
\begin{aligned}
& \sum_{j=1}^{L-1}\left(e^{-v_{j}}-e^{-v_{j+1}}\right)\left[\lambda\left(1-e^{-s_{j}^{\prime}}\right)+\mu\left(1-e^{-s_{j}^{\prime}}\left(1+s_{j}^{\prime}\right)\right)\right] \\
& \approx \frac{\lambda P_{a v}+\mu Q_{a v}}{L-1} \sum_{j=1}^{L-1} \frac{1}{p_{j}}
\end{aligned}
$$

where when $\mu>0, s_{j}^{\prime}=\frac{1}{\mu p_{j}}-\frac{\lambda}{\mu}$, whereas when $\mu=0, s_{j}^{\prime}=$ $\infty$, and (13) simplifies to $c e^{-v_{1}} \approx \frac{P_{a v}}{L-1} \sum_{j=1}^{L-1} v_{j}$ with $v_{j}=$ $\frac{c}{p_{j}}$.

Proof: See Appendix D.

With Lemma 3 and Lemma 4, the main result of this section can be obtained in the following Theorem.

Theorem 1: The asymptotic SU outage probability for a large number of feedback bits is given as, for $\mu>0$,

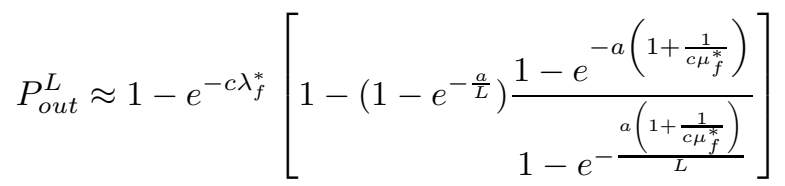

where $a$ is a constant satisfying

$$
\begin{aligned}
& \left(\lambda_{f}^{*} P_{a v}+\mu_{f}^{*} Q_{a v}\right)\left(\lambda_{f}^{*}+\frac{a}{2 c}\right) e^{c \lambda_{f}^{*}} \\
& \approx\left[\left(\lambda_{f}^{*}+\mu_{f}^{*}\right)\left(1-\frac{c \mu_{f}^{*}}{1+c \mu_{f}^{*}}\left(1-e^{-a\left(1+\frac{1}{c \mu_{f}^{*}}\right)}\right)\right)\right. \\
& \left.\quad-\frac{c\left(\mu_{f}^{*}\right)^{2}}{\left(1+c \mu_{f}^{*}\right)^{2}}\left(1-e^{-a\left(1+\frac{1}{c \mu_{f}^{*}}\right)}\left(1+a\left(1+\frac{1}{c \mu_{f}^{*}}\right)\right)\right)\right] .
\end{aligned}
$$


And we have $\lim _{L \rightarrow \infty} P_{\text {out }}^{L}$ we
$e^{-c \lambda_{f}^{*}}\left[1-\frac{1-e^{-a\left(1+\frac{1}{c \mu_{f}^{*}}\right)}}{1+\frac{1}{c \mu_{f}^{*}}}\right]$. For $\mu=0, P_{\text {out }}^{L} \approx$
$1-e^{-c \lambda_{f}^{*}\left(1+\frac{\beta}{L}\right)}$, where $\beta$ is a constant given by
$e^{-c \lambda_{f}^{*}} \approx \lambda_{f}^{*} P_{a v} \frac{e^{\beta}-1}{\beta}$. In this case we also have
$\lim _{L \rightarrow \infty} P_{\text {out }}^{L}=1-e^{-c \lambda_{f}^{*}}$.

Proof: See Appendix E.

\section{QPA FOR Multiple SECONDARY USERS (SUs) \\ SPECTRUM SHARING OVER A COGNITIVE MUltiple ACCESS CHANNEL}

In our system model, when more than one SU transmit to a SU-BS and share the same spectrum with an existing PU, it becomes a cognitive multiple access channel (CMAC) network [28]. Given $M$ SUs, let $g_{1 i}$ and $g_{0 i}$ denote the channel power gain for the link from the $i$-th SU-Tx $(i=$ $1, \ldots, M)$ to the SU-BS and the PU-BS, respectively. With perfect CSI assumption, we indicate the transmit power for SUs as $P\left(\mathbf{g}_{0}, \mathbf{g}_{1}\right) \triangleq\left\{p_{1}\left(\mathbf{g}_{0}, \mathbf{g}_{1}\right), \ldots, p_{M}\left(\mathbf{g}_{0}, \mathbf{g}_{1}\right)\right\}^{T}$, where $\mathbf{g}_{0} \triangleq\left\{g_{01} \ldots, g_{0 M}\right\}^{T}$ and $\mathbf{g}_{1} \triangleq\left\{g_{11} \ldots, g_{1 M}\right\}^{T}$. Then the maximum achievable sum rate for CMAC [28][29] can be expressed as $\hat{R}\left(\mathbf{g}_{0}, \mathbf{g}_{1}\right)=\frac{1}{2} \log \left(1+\mathbf{g}_{1}^{T} P\left(\mathbf{g}_{0}, \mathbf{g}_{1}\right)\right)$. In this case, we consider the sum-rate outage probability of SUs with given $r_{0}$ given by $\hat{P}_{\text {out }}=\operatorname{Pr}\left\{\hat{R}\left(\mathbf{g}_{0}, \mathbf{g}_{1}\right)<r_{0}\right\}$. Thus, the sum-rate outage minimization problem for the CMAC, under a sum ATP of all SUs constraint together with a long term AIP constraint at the PU-BS, can be formulated as

$$
\begin{array}{ll}
\underset{P\left(\mathbf{g}_{0}, \mathbf{g}_{1}\right) \geq 0}{\operatorname{minimize}} & \operatorname{Pr}\left\{\frac{1}{2} \log \left(1+\mathbf{g}_{1}^{T} P\left(\mathbf{g}_{0}, \mathbf{g}_{1}\right)\right)<r_{0}\right\} \\
\text { subject to } & E\left[\sum_{i=1}^{M} p_{i}\left(\mathbf{g}_{0}, \mathbf{g}_{1}\right)\right] \leq P_{a v}, \\
& E\left[\mathbf{g}_{0}^{T} P\left(\mathbf{g}_{0}, \mathbf{g}_{1}\right)\right] \leq Q_{a v} .
\end{array}
$$

Using a similar technique as [28], it is not hard to verify that the optimal transmit power control policy for Problem (15) is given as: $p_{i}^{*}\left(\mathbf{g}_{0}, \mathbf{g}_{1}\right)=$ $\left\{\begin{array}{lc}\frac{c}{g_{1 n}}, & \text { if } i=n \text { and } \hat{\lambda}_{f}^{*}+\hat{\mu}_{f}^{*} g_{0 n}<\frac{g_{1 n}}{c}, \text { where } \\ 0, & \text { otherwise }\end{array}\right.$ $n=\operatorname{argmin}_{\{i=1, \ldots, M\}} \frac{\hat{\lambda}_{f}^{*}+\hat{\mu}_{f}^{*} g_{0 i}}{g_{1 i}}, c=e^{2 r_{0}}-1$, and $\lambda_{f}^{*}, \mu_{f}^{*}$ are the optimal nonnegative Lagrange multipliers associated with the sum ATP constraint and the AIP constraint, respectively.

Remark 5: The above optimal transmission law for CMAC with full CSI implies that if $\min _{\{i=1, \ldots, M\}} \frac{\hat{\lambda}_{f}^{*}+\hat{\mu}_{f}^{*} g_{0 i}}{g_{1 i}}<\frac{1}{c}$, only one SU with the minimum $\frac{\hat{\lambda}_{f}^{*}+\hat{\mu}_{f}^{*} g_{0 i}}{g_{1 i}}$ will transmit, otherwise, none of SUs is allowed to transmit and an outage occurs.

When only quantized CSI is available at the SU transmitters, let $\mathcal{P}=\left\{P_{1}, \ldots, P_{L}\right\}$ with $P_{j}=\left\{p_{1 j}, \ldots, p_{M j}\right\}^{T}$ denote the power codebook and $\left\{\mathcal{R}_{j}, j=1, \ldots L\right\}$ represent the quantization regions. Then with the limited feedback strategy as stated in Section III, the sum-rate outage minimization problem for the CMAC network with finite rate feedback can be expressed as

$$
\begin{array}{ll}
\underset{P_{j} \geq 0, \mathcal{R}_{j} \forall j}{\operatorname{minimize}} & \sum_{j=1}^{L} E\left[\hat{X}_{j} \mid \mathcal{R}_{j}\right] \operatorname{Pr}\left(\mathcal{R}_{j}\right) \\
\text { subject to } & \sum_{j=1}^{L} E\left[\sum_{i=1}^{M} p_{i j} \mid \mathcal{R}_{j}\right] \operatorname{Pr}\left(\mathcal{R}_{j}\right) \leq P_{a v}, \\
& \sum_{j=1}^{L} E\left[\mathbf{g}_{0}^{T} P_{j} \mid \mathcal{R}_{j}\right] \operatorname{Pr}\left(\mathcal{R}_{j}\right) \leq Q_{a v},
\end{array}
$$

where $\hat{X}_{j}, j=1, \ldots, L$, is an indicator function, defined as, in region $\mathcal{R}_{j}, \hat{X}_{j}=1$ if $\frac{1}{2} \log \left(1+\mathbf{g}_{1}^{T} P_{j}\right)<r_{0}$, and 0 otherwise.

It turns out that the structure of the optimal quantization regions for the CMAC case is much more complex than the single SU case. Fig. 3 gives an example of what (a locally) optimal quantization structure looks like by using the SPSA algorithm for $M=2$ with 2 bits of feedback, i.e., 4 quantization regions spanning the entire $\left(\mathbf{g}_{0}, \mathbf{g}_{1}\right)$ space (each color stands for a particular region). Since the quantization structure for two SUs is four-dimensional, in Fig. 3 we plot two of its low-dimensional projections for visual illustration. From Fig. 3, we can clearly see that unlike the single SU case, which possesses a nice "stepwise" quantization regions structure as we derived in Section III, it is extremely difficult to compute the volumes of these high-dimensional regions with irregular shapes in general. Thus, the optimal QPA algorithm (as well as the other algorithms derived based on the analysis of the the optimal QPA algorithm, including the ZPiORA algorithm in Section III-C and the high resolution quantization analysis in Section IV) ${ }^{2}$ designed for the single SU case cannot be directly applied to the CMAC case .

As we mentioned before, the SPSA algorithm is computationally highly complex with a long convergence time, and due to no explicit expression being available for the outage probability of Problem (16), a modified GLA with a Lagrangian distortion measure cannot be directly applied here. However, the GLASFA method introduced in Section III-C with a sigmoid function approximating the indicator function, can be employed to obtain a suboptimal solution for Problem (16). Thus we have $\hat{X}_{j} \approx \sigma\left(k\left(\frac{1}{2} \log \left(1+\mathbf{g}_{1}^{T} P_{j}\right)-r_{0}\right)\right), j=$ $1, \ldots, L$, where $\sigma(x)=\frac{1}{1+e^{x}}$. We can apply the procedure of the GLASFA method stated in Section III-C to solve Problem (16), except that the two optimality conditions of GLASFA method for the CMAC case become the following. Given a random initial power codebook, generate the optimal partition regions by $\mathcal{R}_{j}=\left\{\left(\mathbf{g}_{0}, \mathbf{g}_{1}\right): \hat{X}_{j}+\right.$ $\left.W^{T} P_{j} \leq \hat{X}_{m}+W^{T} P_{m}, \forall m \neq j\right\}, m, j=1, \ldots, L$, where $W=\left\{\left(\lambda+\mu g_{01}\right), \ldots,\left(\lambda+\mu g_{0 M}\right)\right\}^{T}$. Then, given optimal partition regions, update the power codebook by $P_{j} \approx$ $\operatorname{argmin}_{P_{j} \geq 0} E\left[\sigma\left(k\left(\frac{1}{2} \log \left(1+\mathbf{g}_{1}^{T} P_{j}\right)-r_{0}\right)\right)+W^{T} P_{j} \mid R_{j}\right] \operatorname{Pr}\left(R_{j}\right)$ for $j=1, \ldots, L$. Numerical results illustrate that with only 8 bits of feedback, the outage probability performance of the

\footnotetext{
${ }^{2}$ To highlight this issue, we have presented the analysis of the CMAC case in a separate section, namely Section V, where we apply the suboptimal GLASFA algorithm introduced in Section III-C as a possible power allocation algorithm. Note that in Section III-C, GLASFA is used solely for the purpose of comparison with our proposed suboptimal ZPiORA algorithm.
} 


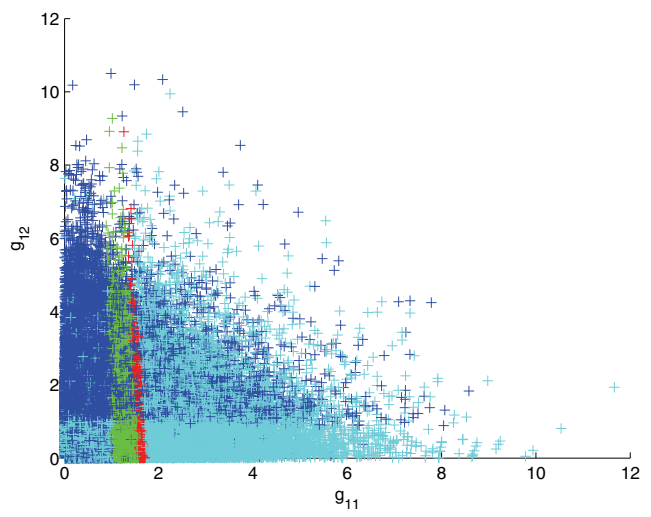

(a) The projection of quantization structure on $\mathbf{g}_{1}$ space.

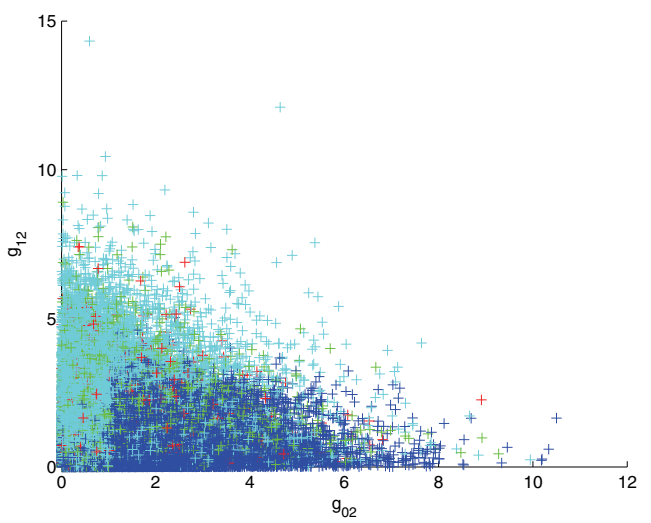

(b) The projection of quantization structure on the coordinate plane of $g_{02}$ and $g_{12}$.

Fig. 3. Structure of quantization regions (spanning over the entire $\left(\mathbf{g}_{0}, \mathbf{g}_{1}\right)$ space) for the case of two SUs with 2 bits of feedback obtained by SPSA algorithm (since the quantization structure is four-dimensional, we only plot its projections on lower dimensions ).

CMAC optimization problem with limited feedback (16) for two SUs using GLASFA can eliminate most of the gap with the corresponding full CSI case. See section VI for more details on simulation results for this algorithm.

\section{Numerical Results}

In this section, we will examine the outage probability performance of the $\mathrm{SU}$ in a narrowband spectrum sharing system with the proposed power allocation strategies via numerical simulations. All the channels involved are assumed to be independent and undergo identical Rayleigh fading, i.e, channel power gain $g_{0}$ and $g_{1}$ are independent and identically exponentially distributed with unity mean. The required transmission rate is taken to be $r_{0}=0.25$ nats per channel use.

Fig. 4 displays the SU outage probability performance of the suboptimal algorithm ZPiORA versus $P_{a v}$ with feedback bits $B=\{1,2\}$, under $Q_{a v}=-5 \mathrm{~dB}$ and $Q_{a v}=0 \mathrm{~dB}$ respectively, and compares these results with the corresponding outage performance of the suboptimal method MEPPR and the optimal QPA. As observed from Fig. 4, when $Q_{a v}=-5$ $\mathrm{dB}$, with $B$ fixed, the outage performances of ZPiORA and corresponding optimal QPA almost overlap with each other.

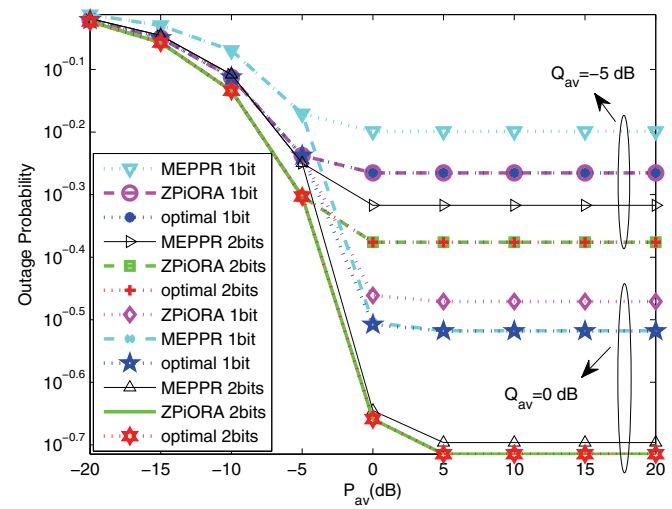

Fig. 4. Outage probability performance comparison between ZPiORA, MEPPR and optimal QPA.

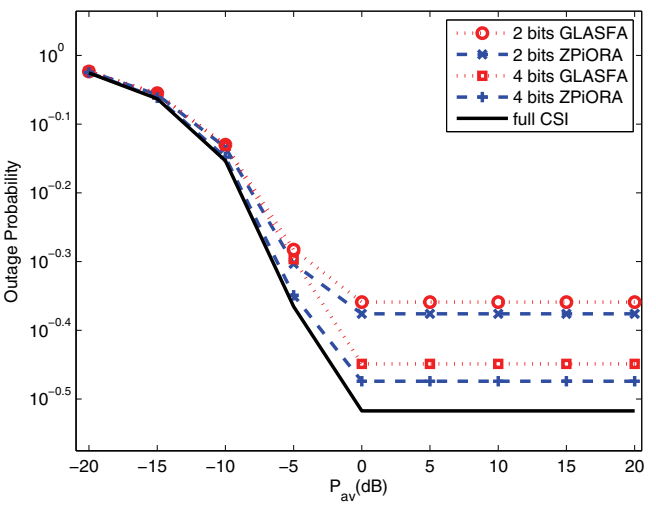

Fig. 5. Outage probability performance comparison between ZPiORA and other possible suboptimal algorithm: GLASFA.

When $Q_{a v}=0 \mathrm{~dB}$ and $P_{a v} \leq-5 \mathrm{~dB}$, with the same number of feedback bits, the outage performances of these two methods are still indistinguishable; and with $P_{a v}>-5 \mathrm{~dB}$, the outage performance gap between ZPiORA and corresponding optimal QPA is decreasing with increasing B. For example, with 1 bit feedback, at $P_{a v}=10 \mathrm{~dB}$, the outage gap between ZPiORA and optimal QPA is 0.0347 , but with 2 bits of feedback, the outage performance of these two methods are very close to each other, which agrees with Lemma 2 that ZPiORA is a near-optimal algorithm for large number of feedback bits. Now we look at the performance comparison between ZPiORA and MEPPR. As illustrated in Fig. 4, when $P_{a v}$ or $Q_{a v}$ is small, with B bits feedback, the performance of ZPiORA is always better than MEPPR. This is attributed to the fact that when $P_{a v}$ or $Q_{a v}$ is small, it can be easily verified that $p_{L}$ is close to zero, but MEPPR always uses $p_{L}>0$. However, when both $P_{a v}$ and $Q_{a v}$ are large (e.g. $P_{a v} \geq 0 \mathrm{~dB}$ and $Q_{a v}=0 \mathrm{~dB}$ ), for 1 bit feedback case, MEPPR outperforms ZPiORA and performs very close to the optimal QPA, whereas with a sufficiently large number of feedback bits (in fact, with more than just 2 bits of feedback), ZPiORA is a more accurate approximation due to Lemma 2. These results confirm the ZPiORA is a better option for a large number of feedback bits, not to mention that ZPiORA is much simpler to implement than MEPPR. 


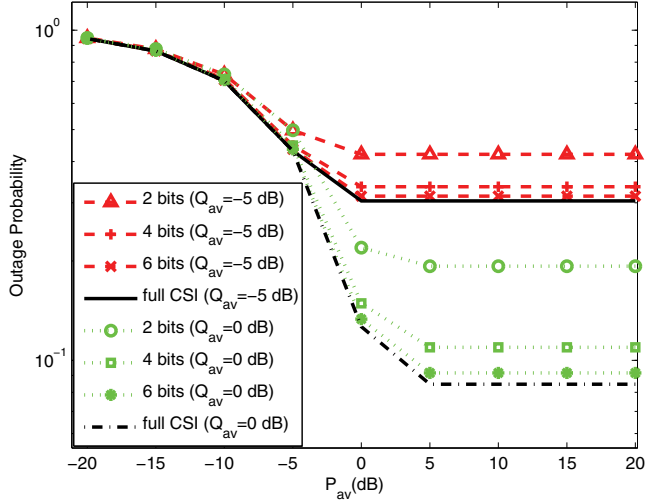

Fig. 6. Effect of increasing feedback bits on outage performance of SU.

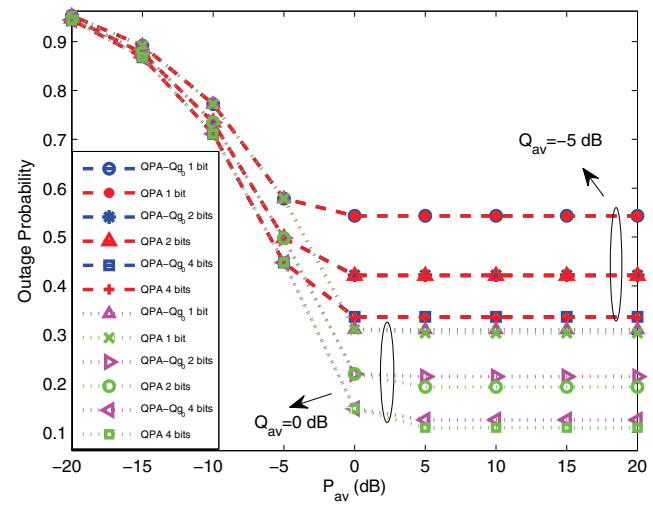

Fig. 7. Outage probability performance of QPA-Q $g_{0}$ algorithm.

In addition, Fig. 5 compares the outage performance of ZPiORA with another suboptimal method (GLASFA) with $Q_{a v}=-5 \mathrm{~dB}$. We can easily observe that with a fixed number of feedback bits ( 2 bits or 4 bits), ZPiORA always outperforms GLASFA. And ZPiORA is also substantially faster than GLASFA. For example, with fixed $\lambda$ and $\mu$ and 4 bits of feedback $\left(Q_{a v}=-5 \mathrm{~dB}, P_{a v}=10 \mathrm{~dB}\right)$, when implemented in MATLAB (version 7.11.0.584 (R2010b)) on a AMD Quad-Core processor (CPU P940 with a clock speed of $1.70 \mathrm{GHz}$ and a memory of $4 \mathrm{~GB}$ ), it was seen that GLASFA (with 100,000 training samples, starting $k=20$ and increasing it by a factor of 1.5 at each step which finally converged at about $k=768.8672$ ) took approximately 299.442522 seconds (different initial guesses of the power codebook may result in different convergence time). In comparison, ZPiORA took only 0.006237 seconds to achieve comparable levels of accuracy. These results further confirm the efficiency of ZPiORA.

Fig. 6 illustrates the outage performance of SU with optimal QPA strategy versus $P_{a v}$ with feedback bits $B=\{2,4,6\}$, under $Q_{a v}=-5 \mathrm{~dB}$ and $Q_{a v}=0 \mathrm{~dB}$ respectively, and studies the effect of increasing the number of feedback bits on the outage performance. For comparison, we also plot the corresponding SU outage performance with full CSI case. Since ZPiORA is an efficient suboptimal method for large number of feedback bits, we employ ZPiORA to obtain the

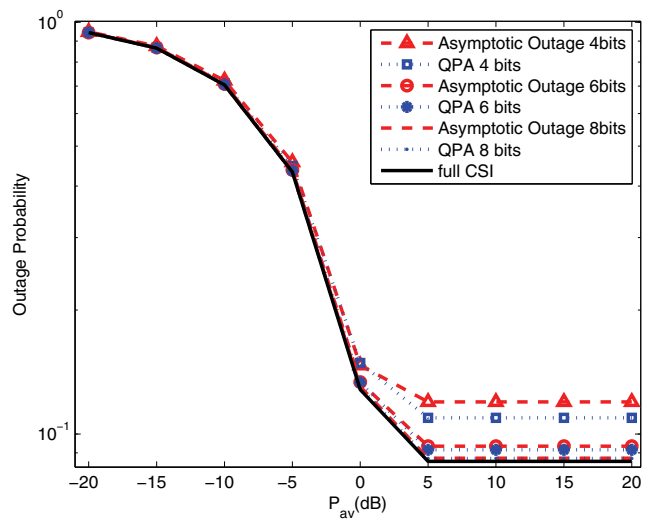

Fig. 8. Comparison between asymptotic outage performance and QPA performance with $Q_{a v}=0 d B$.

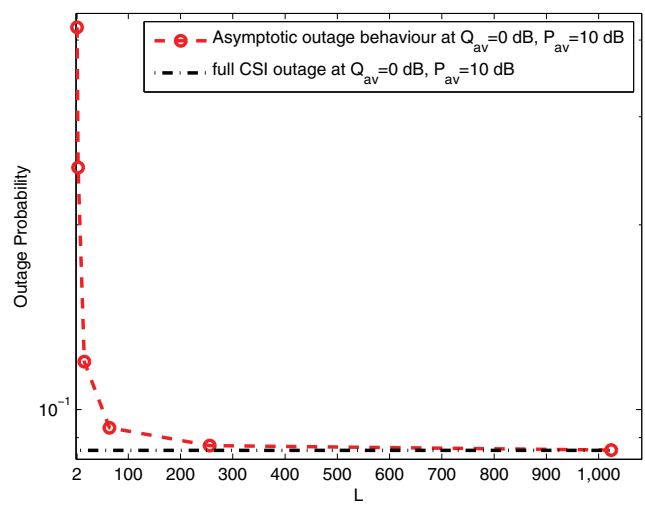

Fig. 9. Asymptotic outage behaviour versus the number of quantization level L.

outage performance instead of using optimal QPA for $B=6$ bits. First, it can be easily observed that all the outage curves decrease gradually as $P_{a v}$ increases until $P_{a v}$ reaches a certain threshold, when the outage probability attains a floor. This is due to the fact that in the high $P_{a v}$ regime, the AIP constraint dominates. For a fixed number of feedback bits, the higher $Q_{a v}$ is, the smaller the resultant outage probability is, since higher $Q_{a v}$ means PU can tolerate more interference. Fig. 6 also illustrates that for fixed $Q_{a v}$, introducing one extra bit of feedback substantially reduces the outage gap between QPA and the perfect CSI case. To be specific, for $Q_{a v}=0 \mathrm{~dB}$ and $P_{a v}=10 \mathrm{~dB}$, with 2 bits, 4 bits and 6 bits of feedback, the outage gaps with the full CSI case are approximately 0.1083 , 0.0249 and 0.006979 respectively. And for any $Q_{a v}$, only 6 bits of feedback seem to result in an SU outage performance very close to that with full CSI case.

Fig. 7 shows the outage performance of QPA-Q $g_{0}$ algorithm, introduced in Section III-B, versus $P_{a v}$ with feedback bits $B=\{1,2,4\}$, under $Q_{a v}=-5 \mathrm{~dB}$ and $Q_{a v}=0 \mathrm{~dB}$ respectively, and compares it with corresponding optimal QPA algorithm. It can be observed from Fig. 7 that optimal QPA algorithm always outperforms QPA-Q $g_{0}$ as expected, however the outage performance gap between these two schemes is considerately small. When $Q_{a v}=-5 \mathrm{~dB}$, with $1,2,4$ bits of feedback, the outage performance of these two algorithms 


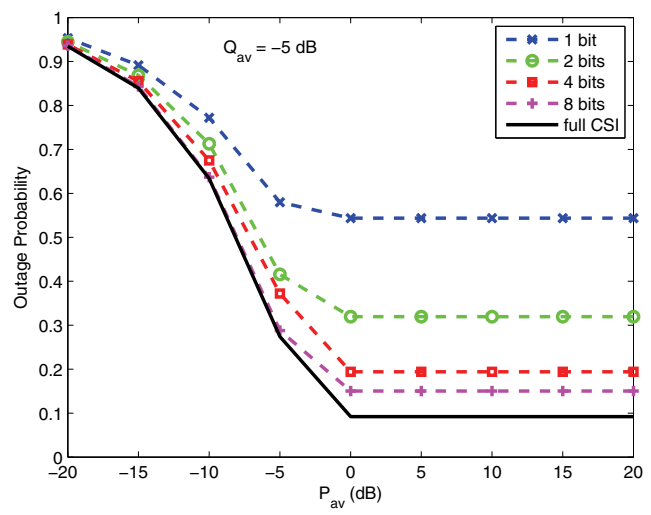

Fig. 10. Outage probability performance of a CMAC with two SUs using GLASFA.

almost overlap with each other. When $Q_{a v}=0 \mathrm{~dB}$, a small outage gap appears between QPA-Q $g_{0}$ and optimal QPA algorithm. For example, with 2 and 4 bits of feedback, at $P_{a v}=10 \mathrm{~dB}$, the outage gaps are only 0.0213 and 0.0160 , respectively.

Fig. 8 compares the asymptotic outage performance derived in Theorem 1 and the optimal QPA performance $B=\{4,6,8\}$ under $Q_{a v}=0 \mathrm{~dB}$. It is clearly observed that increasing number of feedback bits substantially shrinks the outage performance gap between the asymptotic outage approximation and the corresponding optimal QPA performance. For instance, with 4, 6, 8 bits of feedback at $P_{a v}=10 \mathrm{~dB}$, the outage gap between the asymptotic outage approximation and the corresponding optimal QPA are around 0.0325, 0.00618, 0.000168 respectively. These results confirm that the derived asymptotic outage expressions in Theorem 1 are highly accurate for $B \geq 8$ bits of feedback. In addition, Figure 9 depicts the asymptotic outage probability behavior of SU versus the number of quantization level $L$ at $Q_{a v}=0 \mathrm{~dB}, P_{a v}=10$ $\mathrm{dB}$, and compares the result with the corresponding full CSI performance. It can be seen from Fig. 9 that the outage decreases as the number of quantization level $L$ increases, however, as $L$ increases beyond a certain number $\left(L \geq 2^{8}\right.$, i.e, $B \geq 8$ bits), the outage probability curve starts to saturate and approaches the full CSI performance. This further confirms that only a small number of feedback bits is enough to obtain an outage performance close to the perfect CSI-based performance.

Fig. 10 shows the outage performance of CMAC optimization problem (16) with limited feedback solved by the GLASFA, for two SUs (i.e., $M=2$ ) with $Q_{a v}=-5$ $\mathrm{dB}$. The important observation from this figure is that again only a few bits of feedback can remove most of the gap with the corresponding full CSI performance. For example, at $P_{a v}=10 \mathrm{~dB}$, there is only around 0.0584 outage gap between 8 bits of feedback and the full CSI counterpart.

\section{CONCLUSIONS AND EXTENSIONS}

In this paper, we designed optimal power allocation algorithms for secondary outage probability minimization with quantized CSI information for a narrowband spectrum sharing cognitive radio framework under an ATP constraint at SU-TX and an AIP constraint at PU-RX. We prove that the optimal channel partition structure has a "stepwise" pattern based on which an efficient optimal power codebook design algorithm is provided. In the case of a large number of feedback bits, we derive a novel low-complexity suboptimal algorithm ZPiORA which is seen to outperform alternative suboptimal algorithms based on approximations used in the existing literature. We also derive explicit expressions for asymptotic behavior of the SU outage probability for a large number of feedback bits. Although the presented optimal power codebook design methods result in locally optimal solutions (due to the nonconvexity of the quantized power allocation problem), numerical results illustrate that only 6 bits of feedback result in SU outage performance very close to that obtained with full CSI at the SU transmitter. An extended study on multiple SUs (CMAC) is provided as well. Future work will involve extending the results to more complex wideband spectrum sharing scenario along with consideration of other types of interference constraints at the PU receiver.

\section{APPENDIX}

\section{A. Proof of Lemma 1:}

We use an analysis method similar to [27] to prove our problem's optimal quantizer structure. Let $\mathcal{P}=\left\{p_{1}, \ldots, p_{L}\right\}$, where $p_{1}>\cdots>p_{L} \geq 0$, and the corresponding channel partitioning $\mathcal{R}=\left\{\mathcal{R}_{1}, \ldots, \mathcal{R}_{L}\right\}$ denote the optimal solution to the Problem (2), and $p\left(g_{0}, g_{1}\right)=p_{j}$, if $\left(g_{0}, g_{1}\right) \in \mathcal{R}_{j}$.

Let $\mathcal{R}_{j}^{*}=\left\{\left(g_{0}, g_{1}\right): v_{j} \leq g_{1}<v_{j+1}, 0 \leq g_{0}<s_{j}\right\}, j=$ $1, \ldots, L-1$ and $\mathcal{R}_{L}^{*}=\mathcal{R}_{L 1}^{*} \cup \mathcal{R}_{L 2}^{*}=\left\{\left(g_{0}, g_{1}\right): v_{j} \leq\right.$ $\left.g_{1}<v_{j+1}, g_{0} \geq s_{j}, \forall j=0,1, \ldots, L-1\right\} \cup\left\{\left(g_{0}, g_{1}\right):\right.$ $\left.g_{1} \geq v_{L}, g_{0} \geq 0\right\}$, where $s_{0}=0$ and $v_{0}=0$. We assume that the set $\mathcal{R}_{j}^{*} \backslash \mathcal{R}_{j}$ is a non-empty set, where $\backslash$ is the set subtraction operation (i.e, if $\left(g_{0}, g_{1}\right) \in \mathcal{R}_{j}^{*} \backslash \mathcal{R}_{j}$, then $\left(g_{0}, g_{1}\right) \in \mathcal{R}_{j}^{*}$ but $\left.\left(g_{0}, g_{1}\right) \notin \mathcal{R}_{j}\right)$. Then, the set $\mathcal{R}_{j}^{*} \backslash \mathcal{R}_{j}$ can be partitioned into two subsets $S_{j}^{-}=\left(\mathcal{R}_{j}^{*} \backslash \mathcal{R}_{j}\right) \cap\left(\cup_{k=1}^{j-1} \mathcal{R}_{k}\right)$ and $S_{j}^{+}=\left(\mathcal{R}_{j}^{*} \backslash \mathcal{R}_{j}\right) \cap\left(\cup_{k=j+1}^{L} \mathcal{R}_{k}\right)$. In what follows, we denote the empty set by $\emptyset$.

(1): We will show that $S_{j}^{-}=\emptyset, \forall j=1, \ldots, L$.

(a): When $j=1$, it is obvious that $S_{1}^{-}=\emptyset$. When $1<j<L$, if $S_{j}^{-} \neq \emptyset$, then we can always reassign the set $S_{j}^{-}$into region $\mathcal{R}_{j}$ without changing the overall outage probability. This is due to the fact that within the set $S_{j}^{-} \in \mathcal{R}_{j}^{*}$, we have $v_{j} \leq g_{1}<v_{j+1}$ resulting in $\frac{1}{2} \log \left(1+g_{1} p_{j}\right) \geq r_{0}$, and the power level in $\left(\cup_{k=1}^{j-1} \mathcal{R}_{k}\right)$ satisfies $p_{k}>p_{j}$. Thus $S_{j}^{-}$is never in outage. However, the new assignment can achieve a lower Lagrange dual function (LDF) in (3), due to $g^{\prime}(\lambda, \mu)-g(\lambda, \mu)=E\left[\left(\lambda+\mu g_{0}\right)\left(p_{j}-p_{k}\right) \mid S_{j}^{-}\right] \operatorname{Pr}\left(S_{j}^{-}\right)<0$, where $g^{\prime}(\lambda, \mu)$ denotes the LDF with the new assignment, which contradicts the optimality of the solution $\mathcal{P}, \mathcal{R}$.

(b) When $j=L$, if $S_{L}^{-} \neq \emptyset$, we can again reassign the set $S_{L}^{-}$into region $\mathcal{R}_{L}$. 1) If some part of $S_{L}^{-}$is in the set $\left\{\left(g_{0}, g_{1}\right): 0 \leq g_{1}<v_{1}, g_{0} \geq 0\right\}$ of $\mathcal{R}_{L 1}^{*}$, we have $\frac{1}{2} \log \left(1+g_{1} p_{1}\right)<r_{0}$, which implies that this part of $S_{L}^{-}$is always in outage. Therefore, this reassignment for this part of $S_{L}^{-}$will not change the outage probability but will decrease the LDF due to the power level $p_{L}$ in $\mathcal{R}_{L}$ is the lowest. 2) For any $j(j=1, \ldots, L-1)$, if some part of $S_{L}^{-}$(denoted by " $S_{L p}^{-}$") exists in the set $\left\{\left(g_{0}, g_{1}\right): v_{j} \leq g_{1}<v_{j+1}, g_{0} \geq s_{j}\right\}$ 
of $\mathcal{R}_{L 1}^{*}$, we have $\frac{1}{2} \log \left(1+g_{1} p_{j}\right) \geq r_{0}, \frac{1}{2} \log \left(1+g_{1} p_{j+1}\right)<r_{0}$ and $\left(\lambda+\mu g_{0}\right)\left(p_{j}-p_{L}\right) \geq 1$. And given $S_{L p}^{-} \subset\left(\cup_{k=1}^{L-1} \mathcal{R}_{k}\right)$, let the power level for $S_{L p}^{-}$be $p_{k}$ (where $k$ could be any value from $\{1, \ldots, L-1\}$ ). Reassigning this part of set $S_{L}^{-}$ into region $\mathcal{R}_{L}$ will reduce the value of the LDF, since if $k \leq j$ (implying $\left.p_{k} \geq p_{j}\right), g^{\prime}(\lambda, \mu)-g(\lambda, \mu)=E[1+$ $\left.p_{L}\left(\lambda+\mu g_{0}\right)-p_{k}\left(\lambda+\mu g_{0}\right) \mid S_{L p}^{-}\right] \operatorname{Pr}\left(S_{L p}^{-}\right)<0$ and if $k>j$ (implying $\left.p_{k}<p_{j}\right), g^{\prime}(\lambda, \mu)-g(\lambda, \mu)=E\left[1+p_{L}\left(\lambda+\mu g_{0}\right)-\right.$ $\left.1-p_{k}\left(\lambda+\mu g_{0}\right) \mid S_{L p}^{-}\right] \operatorname{Pr}\left(S_{L p}^{-}\right)<0$. 3) If some part of $S_{L}^{-}$ belongs to the set $\mathcal{R}_{L 2}^{*}$, similar to (a), we can show that the new partition for this part of $S_{L}^{-}$does not change the overall outage probability and meanwhile reduces the value of the LDF. These all contradict optimality.

(2): We will now show that the set $S_{j}^{+}=\emptyset, j=1, \ldots, L$. When $j=L$, it's straightforward that $S_{L}^{+}=\emptyset$. When $j<L$, we assume that $S_{j}^{+} \neq \emptyset$. Within the set $S_{j}^{+} \in \mathcal{R}_{j}^{*}$, we have $v_{j} \leq g_{1}<v_{j+1}$, implying $\frac{1}{2} \log \left(1+g_{1} p_{j+1}\right)<r_{0}$, or in other words, $S_{j}^{+} \in\left(\cup_{k=j+1}^{L} \mathcal{R}_{k}\right)$ is in outage. We can reallocate the set $S_{j}^{+}$into region $\mathcal{R}_{j}$. This reassignment not only lowers the outage probability $\left(S_{j}^{+}\right.$with $p_{j}$ will not be in outage) but also lowers the value of the LDF, given by $g^{\prime}(\lambda, \mu)-g(\lambda, \mu)=E\left[\left(\lambda+\mu g_{0}\right)\left(p_{j}-p_{k}\right)-1 \mid S_{j}^{+}\right] \operatorname{Pr}\left(S_{j}^{+}\right) \leq$ $E\left[\left(\lambda+\mu g_{0}\right)\left(p_{j}-p_{L}\right)-1 \mid S_{j}^{+}\right] \operatorname{Pr}\left(S_{j}^{+}\right)<0$, due to $g_{0}<s_{j}=$ $\frac{1}{\mu\left(p_{j}-p_{L}\right)}-\frac{\lambda}{\mu}$. This also contradicts optimality.

Therefore, we have $\mathcal{R}_{j}^{*} \backslash \mathcal{R}_{j}=\emptyset, \forall j=1, \ldots, L$, i.e, $\mathcal{R}_{j}^{*} \subseteq \mathcal{R}_{j}, \forall j=1, \ldots, L$. Since $\cup_{j=1}^{L} \mathcal{R}_{j}^{*}=$ the whole space of $\left(g_{0}, g_{1}\right)=\cup_{j=1}^{L} \mathcal{R}_{j}$, and $\mathcal{R}_{j}^{*} \subseteq \mathcal{R}_{j}, \forall j$, we can obtain that $\mathcal{R}_{j}^{*}=\mathcal{R}_{j}, \forall j=1, \ldots, L$.

\section{B. Proof of Lemma 2:}

We assume that $\lim _{L \rightarrow \infty} p_{L} \neq 0$. Let $\delta=\lim _{L \rightarrow \infty} p_{L}>0$. From the KKT condition (8), we have

$$
\begin{aligned}
& e^{-v_{L}} \frac{c}{p_{L}^{2}}\left(p_{L-1}-p_{L}\right)\left(\lambda+\mu\left(1-e^{-s_{L-1}}\right)\right) \\
& =(\lambda+\mu)\left(P_{\text {out }}^{L}+e^{-v_{L}}\right)+\mu \sum_{j=1}^{L-1}\left(e^{-v_{j}}-e^{-v_{j+1}}\right) e^{-s_{j}} s_{j} \\
& \geq(\lambda+\mu)\left(P_{\text {out }}^{L}+e^{-v_{L}}\right)
\end{aligned}
$$

Let $P_{\text {out }}^{f}$ denote the outage probability with full CSI at SU$\mathrm{TX}$, then we have $P_{\text {out }}^{L} \geq P_{\text {out }}^{f}$ and $\lim _{L \rightarrow \infty} P_{\text {out }}^{L}=P_{\text {out }}^{f}$. Taking the limit $L \rightarrow \infty$ on both sides of (17), we have

$$
\begin{aligned}
& \lim _{L \rightarrow \infty} e^{-v_{L}} \frac{c}{p_{L}^{2}}\left(p_{L-1}-p_{L}\right)\left(\lambda+\mu\left(1-e^{-s_{L-1}}\right)\right) \\
& \geq(\lambda+\mu)\left(P_{\text {out }}^{f}+e^{-\frac{c}{\delta}}\right) \neq 0
\end{aligned}
$$

Given $p_{1}>\cdots>p_{L}>0$, it is clear that the sequence $\left\{p_{j}\right\}, j=1,2, \ldots, L$ is a monotonically decreasing sequence bounded below, therefore it must converge to its greatestlower bound $\delta$, as $L \rightarrow \infty$. Therefore, it can be easily shown that for an arbitrarily small $\epsilon>0$, we can always find a sufficiently large $L$ such that $p_{L-1}-p_{L}<\epsilon$. Thus, as $L \rightarrow \infty,\left(p_{L-1}-p_{L}\right) \rightarrow 0$, which implies when $\mu>0$, $s_{L-1}=\frac{1}{\mu\left(p_{L-1}-p_{L}\right)}-\frac{\lambda}{\mu} \rightarrow \infty$. This implies that

$$
\begin{aligned}
& \lim _{L \rightarrow \infty} e^{-v_{L}} \frac{c}{p_{L}^{2}}\left(p_{L-1}-p_{L}\right)\left(\lambda+\mu\left(1-e^{-s_{L-1}}\right)\right) \\
& =e^{-\frac{c}{\delta}} \frac{c}{\delta^{2}}(\lambda+\mu) \lim _{L \rightarrow \infty}\left(p_{L-1}-p_{L}\right)=0,
\end{aligned}
$$

which is in contradiction with (18). Thus, we must have $\lim _{L \rightarrow \infty} p_{L}=0$.

\section{Proof of Lemma 3:}

As $L \rightarrow \infty$, from Lemma 2, we have $p_{L} \rightarrow 0$. Applying it to Problem (5), we have the KKT conditions as (11).

1) $\mu>0$ : From $s_{j}^{\prime}=\frac{1}{\mu p_{j}}-\frac{\lambda}{\mu}$, we have $p_{j}=\frac{1}{\lambda+\mu s_{j}^{\prime}}$, and we also have $p_{0}=\frac{1}{\lambda+\mu s_{0}^{r}}$. Applying it to (11), the right hand side (RHS) of equation (11) becomes,

$$
\begin{aligned}
R H S & =e^{-v_{j}} \frac{c}{p_{j}^{2}}\left[\frac{\lambda+\mu\left(1-e^{-s_{j-1}^{\prime}}\right)}{\lambda+\mu s_{j-1}^{\prime}}-\frac{\lambda+\mu\left(1-e^{-s_{j}^{\prime}}\right)}{\lambda+\mu s_{j}^{\prime}}\right] \\
& =e^{-v_{j}} \frac{c\left(s_{j-1}^{\prime}-s_{j}^{\prime}\right)}{p_{j}^{2}} \frac{\frac{\lambda+\mu\left(1-e^{-s_{j-1}^{\prime}}\right)}{\lambda+\mu s_{j-1}^{\prime}}-\frac{\lambda+\mu\left(1-e^{-s_{j}^{\prime}}\right)}{\lambda+\mu s_{j}^{\prime}}}{s_{j-1}^{\prime}-s_{j}^{\prime}} .
\end{aligned}
$$

From the mean value theorem (MVT), we have

$$
\begin{aligned}
& \frac{\frac{\lambda+\mu\left(1-e^{-s_{j-1}^{\prime}}\right)}{\lambda+\mu s_{j-1}^{\prime}}-\frac{\lambda+\mu\left(1-e^{-s_{j}^{\prime}}\right)}{\lambda+\mu s_{j}^{\prime}}}{s_{j-1}^{\prime}-s_{j}^{\prime}} \\
& =\frac{-\mu}{\left(\lambda+\mu s^{\prime}\right)^{2}}\left[\lambda\left(1-e^{-s^{\prime}}\right)+\mu\left(1-e^{-s^{\prime}}\left(1+s^{\prime}\right)\right)\right],
\end{aligned}
$$

where $s^{\prime} \in\left[s_{j-1}^{\prime}, s_{j}^{\prime}\right)$. As the number of feedback bits $B=$ $\log _{2} L \rightarrow \infty$, the length of quantization interval on $g_{0}$ axis $\left[s_{j-1}^{\prime}, s_{j}^{\prime}\right), j=1, \ldots, L-1$ approaches zero [24]. Hence (21) becomes,

$$
\begin{aligned}
& \frac{\frac{\lambda+\mu\left(1-e^{-s_{j-1}^{\prime}}\right)}{\lambda+\mu s_{j-1}^{\prime}}-\frac{\lambda+\mu\left(1-e^{-s_{j}^{\prime}}\right)}{\lambda+\mu s_{j}^{\prime}}}{s_{j-1}^{\prime}-s_{j}^{\prime}} \\
& \approx \frac{-\mu}{\left(\lambda+\mu s_{j}^{\prime}\right)^{2}}\left[\lambda\left(1-e^{-s_{j}^{\prime}}\right)+\mu\left(1-e^{-s_{j}^{\prime}}\left(1+s_{j}^{\prime}\right)\right)\right] .
\end{aligned}
$$

Applying (22) to (20), we have $R H S \approx e^{-v_{j}} c \mu\left(s_{j}^{\prime}-\right.$ $\left.s_{j-1}^{\prime}\right)\left[\lambda\left(1-e^{-s_{j}^{\prime}}\right)+\mu\left(1-e^{-s_{j}^{\prime}}\left(1+s_{j}^{\prime}\right)\right)\right]$. Similarly, as $L \rightarrow \infty$, we also have the length of quantization interval on the $g_{1}$ axis $\left[v_{j}, v_{j+1}\right), j=1, \ldots, L-2$ approaches zero, thus from MVT, $e^{-v_{j}}-e^{-v_{j+1}} \approx e^{-v_{j}}\left(v_{j+1}-v_{j}\right)$. Thus the left hand side (LHS) of equation (11) can be approximated as, $L H S \approx e^{-v_{j}}\left(v_{j+1}-v_{j}\right)\left[\lambda\left(1-e^{-s_{j}^{\prime}}\right)+\mu\left(1-e^{-s_{j}^{\prime}}\left(1+s_{j}^{\prime}\right)\right)\right.$. Hence, we have $\forall j=1, \ldots, L-2, v_{j+1}-v_{j} \approx c \mu\left(s_{j}^{\prime}-s_{j-1}^{\prime}\right)$, from which we get $s_{j+1}^{\prime}-s_{j}^{\prime} \approx s_{j}^{\prime}-s_{j-1}^{\prime}, \forall j=1, \ldots, L-2$, namely, $s_{L-1}^{\prime}-s_{L-2}^{\prime} \approx \cdots \approx s_{1}^{\prime}-s_{0}^{\prime}$, since $v_{j}=c \lambda+c \mu s_{j}^{\prime}$.

2) $\mu=0$ : In this case, we have $s_{j}^{\prime}=\infty, j=1, \ldots, L-1$. Thus (11) becomes $e^{-v_{j}}-e^{-v_{j+1}}=e^{-v_{j}} \frac{c}{p_{j}^{2}}\left(p_{j-1}-p_{j}\right)$, where $j=1, \ldots, L-1$ and $p_{0}=\frac{1}{\lambda}$, which can be rewritten as $\frac{1}{v_{j}}\left(e^{-v_{j}}-e^{-v_{j+1}}\right)=\frac{1}{v_{j-1}} e^{-v_{j}}\left(v_{j}-v_{j-1}\right)$, where $v_{0}=\frac{c}{p_{0}}=$ $c \lambda$. Applying MVT into as before, we have $\frac{1}{v_{j}} e^{-v_{j}}\left(v_{j+1}-\right.$ $\left.v_{j}\right) \approx \frac{1}{v_{j-1}} e^{-v_{j}}\left(v_{j}-v_{j-1}\right), \forall j=1, \ldots, L-2$, which yields $\frac{v_{j+1}}{v_{j}} \approx \frac{v_{j}}{v_{j-1}}, \forall j=1, \ldots, L-2$, namely, $\frac{v_{L-1}}{v_{L-2}} \approx \cdots \approx \frac{v_{1}}{v_{0}}$.

This completes the proof for Lemma 3. 


\section{Proof of Lemma 4:}

As $L \rightarrow \infty$, from Lemma 2 , we have $p_{L} \rightarrow 0$. Adding the two equations of (9) together and applying $p_{L} \rightarrow 0$, we have

$$
\begin{aligned}
& \sum_{j=1}^{L-1} p_{j}\left(e^{-v_{j}}-e^{-v_{j+1}}\right)\left[\lambda\left(1-e^{-s_{j}^{\prime}}\right)+\mu\left(1-e^{-s_{j}^{\prime}}\left(1+s_{j}^{\prime}\right)\right)\right] \\
& =\lambda P_{a v}+\mu Q_{a v} .
\end{aligned}
$$

The KKT conditions (11) can be rewritten as

$$
\begin{aligned}
& p_{j}\left(e^{-v_{j}}-e^{-v_{j+1}}\right)\left[\lambda\left(1-e^{-s_{j}^{\prime}}\right)+\mu\left(1-e^{-s_{j}^{\prime}}\left(1+s_{j}^{\prime}\right)\right)\right] \\
& =p_{j-1} e^{-v_{j}}\left(v_{j}-v_{j-1}\right) \frac{\left[\hat{f}^{\prime}\left(p_{j-1}\right)-\hat{f}^{\prime}\left(p_{j}\right)\right]}{p_{j-1}-p_{j}}
\end{aligned}
$$

where $\hat{f}^{\prime}\left(p_{j}\right)=p_{j}\left(\lambda+\mu\left(1-e^{-s_{j}^{\prime}}\right)\right)$. As mentioned before, when $L \rightarrow \infty$, we have the length of quantization interval on the $g_{1}$ axis $\left[v_{j-1}, v_{j}\right), j=2, \ldots, L-1$ approaching zero. Hence we also have the length of the interval $\left[p_{j-1}, p_{j}\right), j=$ $2, \ldots, L-1$ approaching zero, since $v_{j}=\frac{c}{p_{j}}$. Thus from MVT, we have

$$
\begin{aligned}
e^{-v_{j-1}}-e^{-v_{j}} & \approx e^{-v_{j}}\left(v_{j}-v_{j-1}\right) \\
\frac{\hat{f}^{\prime}\left(p_{j-1}\right)-\hat{f}^{\prime}\left(p_{j}\right)}{p_{j-1}-p_{j}} & \approx \lambda\left(1-e^{-s_{j-1}^{\prime}}\right)+\mu\left(1-e^{-s_{j-1}^{\prime}}\left(1+s_{j-1}^{\prime}\right)\right) .
\end{aligned}
$$

Applying (25) into (24), we can obtain, $\forall j=2, \ldots, L-1$

$p_{j}\left(e^{-v_{j}}-e^{-v_{j+1}}\right)\left[\lambda\left(1-e^{-s_{j}^{\prime}}\right)+\mu\left(1-e^{-s_{j}^{\prime}}\left(1+s_{j}^{\prime}\right)\right)\right] \approx$

$p_{j-1}\left(e^{-v_{j-1}}-e^{-v_{j}}\right)\left[\lambda\left(1-e^{-s_{j-1}^{\prime}}\right)+\mu\left(1-e^{-s_{j-1}^{\prime}}\left(1+s_{j-1}^{\prime}\right)\right)\right]$.

Then applying the result of (26) into (23), we can have $j=$ $1, \ldots, L-1$

$$
\begin{aligned}
& p_{j}\left(e^{-v_{j}}-e^{-v_{j+1}}\right)\left[\lambda\left(1-e^{-s_{j}^{\prime}}\right)+\mu\left(1-e^{-s_{j}^{\prime}}\left(1+s_{j}^{\prime}\right)\right)\right] \\
& \approx \frac{\lambda P_{a v}+\mu Q_{a v}}{L-1}
\end{aligned}
$$

which gives,

$$
\begin{aligned}
& \sum_{j=1}^{L-1}\left(e^{-v_{j}}-e^{-v_{j+1}}\right)\left[\lambda\left(1-e^{-s_{j}^{\prime}}\right)+\mu\left(1-e^{-s_{j}^{\prime}}\left(1+s_{j}^{\prime}\right)\right)\right] \\
& \approx \frac{\lambda P_{a v}+\mu Q_{a v}}{L-1} \sum_{j=1}^{L-1} \frac{1}{p_{j}} .
\end{aligned}
$$

This completes the proof for Lemma 4.

\section{E. Proof of Theorem 1:}

1) $\mu>0$ : From Lemma 3 , we can easily obtain, $s_{j}^{\prime} \approx$ $j s_{1}^{\prime}, \quad \frac{1}{p_{j}}=\lambda+\mu s_{j}^{\prime} \approx \lambda+j \mu s_{1}^{\prime}$, and $v_{j}=\frac{c}{p_{j}} \approx$ $c \lambda+j c \mu s_{1}^{\prime}, \forall j=1, \ldots, L-1$, Let $z=\sum_{j=1}^{L-1}\left(e^{-v_{j}}-\right.$ $\left.e^{-v_{j+1}}\right)\left[\lambda\left(1-e^{-s_{j}^{\prime}}\right)+\mu\left(1-e^{-s_{j}^{\prime}}\left(1+s_{j}^{\prime}\right)\right)\right]$, which implies that $0<z<\lambda+\mu$. Then from Lemma 4, we have $\frac{1}{L-1} \sum_{j=1}^{L-1} \frac{1}{p_{j}} \approx z^{\prime}$, where $z^{\prime}=\frac{z}{\lambda P_{a v}+\mu Q_{a v}}$ and $0<$ $z^{\prime}<\frac{\lambda+\mu}{\lambda P_{a v}+\mu Q_{a v}}$. Using the above results, we get $s_{1}^{\prime} \approx$ $\frac{2\left(z^{\prime}-\lambda\right)}{\mu L}=\frac{d}{L}$, where $d=\frac{2\left(z^{\prime}-\lambda\right)}{\mu}$. Let $a=c \mu d=2\left(z^{\prime}-\lambda\right) c$, then $s_{1}^{\prime} \approx \frac{a}{c \mu L}$. Since $0<z^{\prime}<\frac{\lambda+\mu}{\lambda P_{a v}+\mu Q_{a v}}$, we have $\lim _{L \rightarrow \infty} \frac{a}{L}=0$. From the definition of $z$ above, we have

$$
\begin{aligned}
z= & (\lambda+\mu) e^{-v_{1}}-\sum_{j=1}^{L-1}\left(e^{-v_{j}}-e^{-v_{j}+1}\right)\left[(\lambda+\mu) e^{-s_{j}^{\prime}}\right. \\
& +\mu e^{\left.-s_{j}^{\prime} s_{j}^{\prime}\right]} \\
\approx & e^{-c \lambda}\left[(\lambda+\mu) e^{-\frac{a}{L}}-\left(1-e^{-\frac{a}{L}}\right)(\lambda+\mu) \sum_{j=1}^{L-1} e^{-j\left(\frac{a}{L}+s_{1}^{\prime}\right)}\right. \\
& -\left(1-e^{\left.-\frac{a}{L}\right) \mu s_{1}^{\prime}} \sum_{j=1}^{L-1} j e^{-j\left(\frac{a}{L}+s_{1}^{\prime}\right)}\right] \\
\approx & e^{-c \lambda}\left[(\lambda+\mu) e^{-\frac{a}{L}}-\left(1-e^{-\frac{a}{L}}\right)(\lambda+\mu) \sum_{j=1}^{L-1} e^{-j \frac{b}{L}}\right. \\
& -\left(1-e^{\left.-\frac{a}{L}\right)} \frac{a}{c L} \sum_{j=1}^{L-1} j e^{-j \frac{b}{L}}\right]
\end{aligned}
$$

where $b=a+L s_{1}^{\prime}=a\left(1+\frac{1}{c \mu}\right)$ and we also have $\lim _{L \rightarrow \infty} \frac{b}{L}=0$. Since $\sum_{j=1}^{L-1} e^{-j \frac{b}{L}}=\frac{1-e^{-b}}{1-e^{-\frac{b}{L}}}-1$, and $\sum_{j=1}^{L-1} j e^{-j \frac{b}{L}}=-\frac{e^{\left(-\frac{b}{L}-b\right)}\left(L e^{\frac{b}{L}}-e^{b}-L+1\right)}{\left(1-e^{-\frac{b}{L}}\right)^{2}}$, (29) becomes

$$
\begin{aligned}
z \approx & e^{-c \lambda}\left[(\lambda+\mu)\left(1-\left(1-e^{-\frac{a}{L}}\right) \frac{1-e^{-b}}{1-e^{-\frac{b}{L}}}\right)\right. \\
& \left.-\left(1-e^{-\frac{a}{L}}\right) \frac{a}{c L} \frac{e^{-\frac{b}{L}}\left(1-e^{-b}\right)-L e^{-b}\left(1-e^{-\frac{b}{L}}\right)}{\left(1-e^{-\frac{b}{L}}\right)^{2}}\right] .
\end{aligned}
$$

Since $\lim _{L \rightarrow \infty} \frac{a}{L}=0$ and $\lim _{L \rightarrow \infty} \frac{b}{L}=0$, we have $1-$ $e^{-\frac{a}{L}} \approx \frac{a}{L}$ and $1-e^{-\frac{b}{L}} \approx \frac{b}{L}$. And when $L \rightarrow \infty$, we approach the full CSI scenario, thus implying $\lambda \approx \lambda^{f}, \mu \approx \mu^{f}$. Using these results in (30), we have

$$
\begin{gathered}
z \approx e^{-c \lambda_{f}^{*}}\left[\left(\lambda_{f}^{*}+\mu_{f}^{*}\right)\left(1-\frac{a}{b}\left(1-e^{-b}\right)\right)\right. \\
\left.-\frac{a^{2}}{c b^{2}}\left(\left(1-\frac{b}{L}\right)\left(1-e^{-b}\right)-b e^{-b}\right)\right] \\
\approx e^{-c \lambda_{f}^{*}}\left[\left(\lambda_{f}^{*}+\mu_{f}^{*}\right)\left(1-\frac{a}{b}\left(1-e^{-b}\right)\right)\right. \\
\left.-\frac{a^{2}}{c b^{2}}\left(1-e^{-b}(1+b)\right)\right] .
\end{gathered}
$$

Since $z=\left(\lambda P_{a v}+\mu Q_{a v}\right) z^{\prime}=\left(\lambda P_{a v}+\mu Q_{a v}\right)\left(\lambda+\frac{a}{2 c}\right) \approx$ $\left(\lambda_{f}^{*} P_{a v}+\mu_{f}^{*} Q_{a v}\right)\left(\lambda_{f}^{*}+\frac{a}{2 c}\right)$, we can obtain $a$ from the following approximation:

$$
\begin{aligned}
& \left(\lambda_{f}^{*} P_{a v}+\mu_{f}^{*} Q_{a v}\right)\left(\lambda_{f}^{*}+\frac{a}{2 c}\right) e^{c \lambda_{f}^{*}} \\
& \approx\left[\left(\lambda_{f}^{*}+\mu_{f}^{*}\right)\left(1-\frac{c \mu_{f}^{*}}{1+c \mu_{f}^{*}}\left(1-e^{-a\left(1+\frac{1}{c \mu_{f}^{*}}\right)}\right)\right)\right. \\
& \left.\quad-\frac{c\left(\mu_{f}^{*}\right)^{2}}{\left(1+c \mu_{f}^{*}\right)^{2}}\left(1-e^{-a\left(1+\frac{1}{c \mu_{f}^{*}}\right)}\left(1+a\left(1+\frac{1}{c \mu_{f}^{*}}\right)\right)\right)\right] .
\end{aligned}
$$


From (32), with given $P_{a v}$ and $Q_{a v}, a$ is a constant. Then when $L$ is large,

$$
\begin{aligned}
P_{\text {out }}^{L} & \approx 1-e^{-v_{1}}+\sum_{j=1}^{L-1}\left(e^{-v_{j}}-e^{-v_{j+1}}\right) e^{-s_{j}^{\prime}} \\
& \approx 1-e^{-c \lambda}\left[e^{-\frac{a}{L}}-\left(1-e^{-\frac{a}{L}}\right) \sum_{j=1}^{L-1} e^{-j \frac{b}{L}}\right] \\
& =1-e^{-c \lambda}\left[1-\left(1-e^{-\frac{a}{L}}\right) \frac{1-e^{-b}}{1-e^{-\frac{b}{L}}}\right] \\
& \approx 1-e^{-c \lambda_{f}^{*}}\left[1-\left(1-e^{-\frac{a}{L}}\right) \frac{1-e^{-a\left(1+\frac{1}{c \mu_{f}^{*}}\right)}}{\left.1-e^{-\frac{a\left(1+\frac{1}{c \mu_{f}^{*}}\right)}{L}}\right],}\right.
\end{aligned}
$$

and $\lim _{L \rightarrow \infty} P_{\text {out }}^{L}=1-e^{-c \lambda_{f}^{*}}\left[1-\frac{1-e^{-a\left(1+\frac{1}{c \mu_{f}^{*}}\right)}}{1+\frac{1}{c \mu_{f}^{*}}}\right]$.

2) $\mu=0$ : Let $y=\frac{v_{1}}{v_{0}}=\frac{v_{1}}{c \lambda}>1$, then again, from Lemma 3 , we can get for $j=1, \ldots, L-1, v_{j} \approx c \lambda y^{j}$. From Lemma 4, we have $e^{-c \lambda y} \approx \frac{\lambda P_{a v}}{L-1} \sum_{j=1}^{L-1} y^{j}=\frac{\lambda P_{a v}}{L-1} \frac{y^{L}-y}{y-1}$. With $x=y-1$, we have, $e^{-c \lambda(1+x)} \approx \lambda P_{a v}(1+x) \frac{(1+x)^{L-1}-1}{x(L-1)}$. Now, suppose $\lim _{L \rightarrow \infty} x L=\infty$. Since $(1+x)^{L-1}>1+(L-$ 1) $x+\frac{1}{2}(L-2)(L-1) x^{2}$, we have $\lim _{L \rightarrow \infty} \frac{(1+x)^{L-1}-1}{(L-1) x}>$ $\lim _{L \rightarrow \infty} 1+\frac{1}{2}(L-2) x=\infty$. Then taking the limit as $L \rightarrow \infty$, we have $\lim _{L \rightarrow \infty} e^{-c \lambda(1+x)}=\infty$, which contradicts $\lim _{L \rightarrow \infty} e^{-c \lambda(1+x)}<1$, thus we must have $\lim _{L \rightarrow \infty} x L=$ $0 \leq \beta<\infty$ (where $\beta$ is a constant), implying as $L \rightarrow \infty$, $x \rightarrow \frac{\beta}{L}$. Applying this result, we get $e^{-c \lambda\left(1+\frac{\beta}{L}\right)} \approx \lambda P_{a v}(1+$ $\left.\frac{\beta}{L}\right) \frac{\left(1+\frac{\beta}{L}\right)^{L-1}-1}{\frac{\beta}{L}(L-1)}$. After taking the limit as $L \rightarrow \infty$ on both sides of above equation, we have $e^{-c \lambda_{f}^{*}} \approx \lambda_{f}^{*} P_{a v} \frac{e^{\beta}-1}{\beta}$, from which one can solve for $\beta$ approximately. Note that in the above approximation, we have used $\lim _{L \rightarrow \infty}\left(1+\frac{\beta}{L}\right)^{L-1}=e^{\beta}$ and when $L$ is large, $\lambda \approx \lambda_{f}^{*}$. Therefore, when $L$ is large, $P_{\text {out }}^{L}=1-e^{-v_{1}}=1-e^{-c \lambda(1+x)} \approx 1-e^{-c \lambda_{f}^{*}\left(1+\frac{\beta}{L}\right)}$, $\lim _{L \rightarrow \infty} P_{\text {out }}^{L}=1-e^{-c \lambda_{f}^{*}}$.

This completes the proof for Theorem 1 .

\section{REFERENCES}

[1] J.M. Peha, "Sharing spectrum through spectrum policy reform and cognitive radio," Proc. IEEE, vol. 97, no. 4, pp. 708-719, Apr. 2009.

[2] J. Mitola III, "Cognitive radio for flexible mobile multimedia communications," in Proc. 1999 IEEE Int. Workshop Mobile Multimedia Commun., pp. 3-10.

[3] A. Goldsmith, S.A. Jafar, I. Maric, and S. Srinivasa, "Breaking spectrum gridlock with cognitive radios: an information theoretic perspective," Proc. IEEE, vol. 97, no. 5, pp. 894-914, May 2009.

[4] X. Kang, Y. Liang, A. Nallanathan, H.K. Garg, and R. Zhang, "Optimal power allocation for fading channels in cognitive radio networks: ergodic capacity and outage capacity," IEEE Trans. Wireless Commun., vol. 8, no. 2, pp. 940-950, Feb. 2009.

[5] A. Ghasemi and E. S. Sousa, "Fundamental limits of spectrum-sharing in fading environments," IEEE Trans. Wireless Commun., vol. 6, no. 2, pp. 649-658, Feb. 2007.

[6] H. A. Suraweera, J. Gao, P. J. Smith, M. Shafi, and M. Faulkner, "Channel capacity limits of cognitive radio in asymmetric fading environments," in Proc. 2008 IEEE International Conf. Commun., pp. 40484053.

[7] L. Musavian and S. Aissa, "Capacity and power allocation for spectrumsharing communications in fading channels," IEEE Trans. Wireless Commun., vol. 8, no. 1, pp. 148-156, Jan. 2009.
[8] L. Musavian and S. Aissa, "Fundamental capacity limits of cognitive radio in fading environments with imperfect channel information," IEEE Trans. Commun., vol. 57, no. 11, pp. 3472-3480, Nov. 2009.

[9] H.A. Suraweera, P.J. Smith, and M. Shafi, "Capacity limits and performance analysis of cognitive radio with imperfect channel knowledge," IEEE Trans. Veh. Technol., vol. 59, no. 4, pp. 1811-1822, Feb. 2010.

[10] K. Huang and R. Zhang, "Cooperative feedback for multi-antenna cognitive radio networks," IEEE Trans. Signal Process., vol. 59, no. 2, pp. 747-758, Feb. 2011.

[11] L. Zhang, Y.-C. Liang, Y. Xin, and H.V. Poor, "Robust cognitive beamforming with partial channel state information," IEEE Trans. Wireless Commun., vol. 8, no. 8, pp. 4143-4153, Aug. 2009.

[12] A.G. Marques, X. Wang, and G.B. Giannakis, "Dynamic resource management for cognitive radios using limited-rate feedback," IEEE Trans. Signal Process., vol. 57, no. 9, pp. 3651-3666, Sep. 2009.

[13] Y. Y. He and S. Dey, "Power allocation in spectrum sharing cognitive radio networks with quantized channel information," IEEE Trans. Commun., vol. 59, no. 6, pp. 1644-1656, June 2011,

[14] M. M. Abdallah, A. H. Salem, M. Alouini, and K. A. Qaraqe, "Adaptive discrete rate and power transmission for spectrum sharing systems," IEEE Trans. Wireless Commun., vol. 11, no. 4, pp. 12831289, Apr. 2012.

[15] S. Huang, X. Liu, and Z. Ding, "Decentralized cognitive radio control based on inference from primary link control information," IEEE J. Sel. Areas Commun., vol. 29, no. 2, Feb. 2011.

[16] K. Eswaran, M. Gastpar, and K. Ramachandran, "Cognitive radio through primary control feedback," IEEE J. Sel. Areas Commun., vol. 29, no. 2, Feb. 2011.

[17] D. Gesbert, S. Hanly, H. Huang, S. S. Shitz, O. Simeone, and W. Yu, "Multi-cell MIMO cooperative networks: a new look at interference," IEEE J. Sel. Areas Commun., vol. 28, no. 9, pp. 1-29, Dec. 2010.

[18] R. Irmer, H. Droste, P. Marsch, M. Grieger, G. Fettweis, S. Brueck, H.-P. Mayer, L. Thiele, and V. Jungnickel, "Coordinated multipoint: concepts, performance, and field trial results," IEEE Commun. Mag., vol. 49, no. 2, pp. 102-111, Feb. 2011.

[19] V. Jungnickel, L. Thiele, M. Schellmann, T. Wirth, T. Haustein, O. Koch, W. Zirwas, and E. Schulz, "Interference-aware scheduling in the multiuser MIMO-OFDM downlink," IEEE Commun. Mag., vol. 47, no. 6, pp. 56-66, June 2009.

[20] A.T. Hoang, Y.-C. Liang, and M. H. Islam, "Power control and channel allocation in cognitive radio networks with primary users' cooperation," IEEE Trans. Mobile Comput., vol. 9, no. 3, pp. 348-360, Mar. 2010.

[21] W. Su, J.D. Matyjas, and S. Batalama, "Active cooperation between primary users and cognitive radio users in heterogeneous ad-Hoc networks," IEEE Trans. Signal Process., vol. 60, no. 4, pp. 1796-1805, Apr. 2012.

[22] R. Zhang, "On peak versus average interference power constraints for protecting primary users in cognitive radio networks," IEEE Trans. Wireless Commun., vol. 8, no. 4, pp. 2112-2120, Apr. 2009.

[23] J. C. Spall, "Implementation of the simultaneous perturbation algorithm for stochastic optimization," IEEE Trans. Aerospace Electron. Syst., vol. 34, no. 3, pp. 817-823, July 1998.

[24] A. Khoshnevis and A. Sabharwal, "Performance of quantized power control in multiple antenna systems," in Proc. 2004 IEEE International Conf. Commun., pp. 803-807.

[25] B. Khoshnevis and Wei Yu, "Joint power control and beamforming codebook design for MISO channels with limited feedback," in Proc. 2009 IEEE Global Telecommun. Conf., pp. 1-6.

[26] P. A. Chou, T. Lookabaugh, and R. M. Gray, "Entropy-constrained vector quantization," IEEE Trans. Acoustics, Speech, Signal Process., vol. 31, no. 1, pp. 31-42, Jan. 1989.

[27] M. A. Khojastepour, G. Yue, X. Wang, and M. Madihian, "Optimal power control in MIMO systems with quantized feedback," IEEE Trans. Wireless Commun., vol. 7, no. 12, pp. 4859-4866, Dec. 2008.

[28] X. Kang, Y.-C. Liang, and H. K. Garg, "Fading cognitive multiple access channels: outage capacity regions and optimal power allocation," IEEE Trans. Wireless Commun., vol. 9, no. 7, pp. 2382-2391, July 2010.

[29] R. Zhang, S. Cui, and Y. Liang, "On ergodic sum capacity of fading cognitive multiple-access and broadcast channels," IEEE Trans. Inf. Theory, vol. 55, no. 11, pp. 5161-5178, Nov. 2009.

[30] S. Sorooshyari, C. W. Tan, and M. Chiang, "Power control for cognitive radio networks: axioms, algorithms, and analysis," IEEE/ACM Trans. Netw., vol. 20, no. 3, pp. 878-891, June 2012.

[31] Y. Shi, Y. T. Hou, and H. Zhou, "Per-node based optimal power control for multi-hop cognitive radio networks," IEEE Trans. Wireless Commun., vol. 8, no. 10, pp. 5290-5299, Oct. 2009.

[32] P. Xia and G. B. Giannakis, "Design and analysis of transmitbeamforming based on limited-rate feedback," IEEE Trans. Signal 
Process., vol. 54, no. 5, pp. 1853-1863, May 2006.

[33] Y. Y. He and S. Dey, "Outage minimization in cognitive radio networks with limited feedback," in Proc. 2012 Australian Commun. Theory Workshop, pp. 84-89.

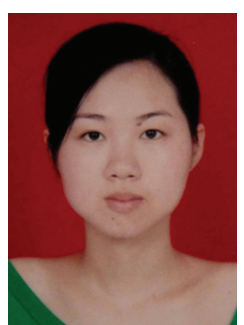

Yuanyuan He (S'08, M'12) received her B.Eng. degree in communication engineering from Yanshan University, China, in 2005, the M.Eng. degree in electronic engineering from Harbin Institute of Technology, China, in 2007, and the Ph.D. degree in the Department of Electrical and Electronic Engineering, University of Melbourne, Melbourne, Australia, in 2011, where she is currently a Postdoctoral Research Fellow since 2011. Her research interests lie in the area of wireless communications limited feedback.

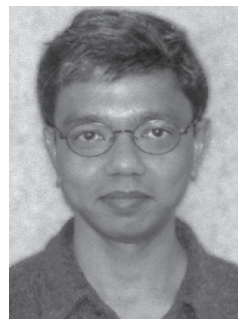

Subhrakanti Dey (SM'06) was born in India in 1968. He received the B.Tech. and M.Tech. degrees from the Department of Electronics and Electrical Communication Engineering, Indian Institute of Technology, Kharagpur, India, in 1991 and 1993, respectively, and the Ph.D. degree from the Department of Systems Engineering, Research School of Information Sciences and Engineering, Australian National University, Canberra, Australia, in 1996.

He has been with the Department of Electrical and Electronic Engineering, University of Melbourne, Parkville, Australia, since February 2000, where he is currently a full Professor. From September 1995 to September 1997 and September 1998 to February 2000, he was a postdoctoral Research Fellow with the Department of Systems Engineering, Australian National University. From September 1997 to September 1998, he was a post-doctoral Research Associate with the Institute for Systems Research, University of Maryland, College Park. His current research interests include networked control systems, wireless communications and networks, signal processing for sensor networks, and stochastic and adaptive estimation and control.

Prof. Dey currently serves on the Editorial Board of Elsevier Systems and Control Letters. He was also an Associate Editor for the IEEE TRANSactions on Signal Processing and the IEEE TRANSACTIONS ON Automatic Control. He is a Senior Member of IEEE. 